\title{
The Young Substellar Companion ROXs 12 B: Near-infrared Spectrum, System Architecture, and Spin-Orbit Misalignment*
}

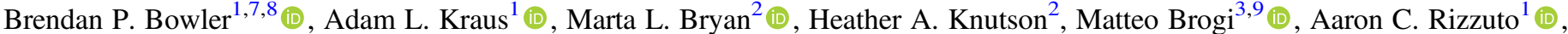 \\ Gregory N. Mace ${ }^{1}$, Andrew Vanderburg ${ }^{4,10}$ (1), Michael C. Liu ${ }^{5}$ (B), Lynne A. Hillenbrand ${ }^{2}$, and Lucas A. Cieza ${ }^{6}$ (1) \\ ${ }^{1}$ McDonald Observatory and the Department of Astronomy, University of Texas at Austin, Austin, TX 78712, USA; bpbowler@astro.as.utexas.edu \\ ${ }^{2}$ California Institute of Technology, 1200 E. California Boulevard, Pasadena, CA 91125, USA \\ ${ }^{3}$ Center for Astrophysics and Space Astronomy, University of Colorado at Boulder, Boulder, CO 80309, USA \\ ${ }^{4}$ Harvard-Smithsonian Center for Astrophysics, Cambridge, MA 02138, USA \\ ${ }^{5}$ Institute for Astronomy, University of Hawai'i i at Mānoa, 2680 Woodlawn Drive, Honolulu, HI 96822, USA \\ ${ }^{6}$ Núcleo de Astronomía, Facultad de Ingeniería y Ciencias, Universidad Diego Portales, Av. Ejercito 441, Santiago, Chile \\ Received 2017 June 30; revised 2017 August 21; accepted 2017 August 24; published 2017 September 28
}

\begin{abstract}
ROXs 12 (2MASS J16262803-2526477) is a young star hosting a directly imaged companion near the deuteriumburning limit. We present a suite of spectroscopic, imaging, and time-series observations to characterize the physical and environmental properties of this system. Moderate-resolution near-infrared spectroscopy of ROXs 12 B from Gemini-North/NIFS and Keck/OSIRIS reveals signatures of low surface gravity including weak alkali absorption lines and a triangular $H$-band pseudocontinuum shape. No signs of $\mathrm{Pa} \beta$ emission are evident. As a population, however, we find that about half $(46 \% \pm 14 \%)$ of young $(\lesssim 15 \mathrm{Myr})$ companions with masses $\lesssim 20 M_{\text {Jup }}$ possess actively accreting subdisks detected via $\mathrm{Pa} \beta$ line emission, which represents a lower limit on the prevalence of circumplanetary disks in general, as some are expected to be in a quiescent phase of accretion. The bolometric luminosity of the companion and age of the host star $\left(6_{-2}^{+4} \mathrm{Myr}\right)$ imply a mass of $17.5 \pm 1.5 M_{\mathrm{Jup}}$ for ROXs $12 \mathrm{~B}$ based on hot-start evolutionary models. We identify a wide (5100 au) tertiary companion to this system, 2MASS J16262774-2527247, that is heavily accreting and exhibits stochastic variability in its $K 2$ light curve. By combining $v \sin i_{*}$ measurements with rotation periods from $K 2$, we constrain the line-of-sight inclinations of ROXs $12 \mathrm{~A}$ and 2MASS J16262774-2527247 and find that they are misaligned by $60_{-11}^{+7}$. In addition, the orbital axis of ROXs $12 \mathrm{~B}$ is likely misaligned from the spin axis of its host star, ROXs $12 \mathrm{~A}$, suggesting that ROXs $12 \mathrm{~B}$ formed akin to fragmenting binary stars or in an equatorial disk that was torqued by the wide stellar tertiary.
\end{abstract}

Key words: brown dwarfs - planetary systems - planets and satellites: atmospheres -

stars: individual (ROXs 12, 2MASS J16262774-2527247) - stars: low-mass

\section{Introduction}

A growing number of directly imaged planetary-mass companions (PMCs; $\lesssim 13 M_{\text {Jup }}$ ) spanning orbital distances of hundreds to thousands of au have been discovered over the past decade through adaptive optics imaging and seeing-limited common proper-motion searches (e.g., Ireland et al. 2011; Bailey et al. 2014; Kraus et al. 2014; Naud et al. 2014; Deacon et al. 2016; Bowler et al. 2017). The origin of this remarkable population is under debate but probably differs from that of giant planets located at smaller separations within $\sim 10$ au. Planet scattering to large separations (Boss 2006; Gotberg et al. 2016), a binary-like formation scenario via turbulent fragmentation (Low \& Lynden-Bell 1976; Boss 2001; Bate 2009), and instabilities in massive protoplanetary disks (e.g., Boss 1997; Kratter \& Lodato 2016) have all been proposed to explain their existence. Unfortunately, robust tests

\footnotetext{
* Some of the data presented herein were obtained at the W. M. Keck Observatory, which is operated as a scientific partnership among the California Institute of Technology, the University of California, and the National Aeronautics and Space Administration. The observatory was made possible by the generous financial support of the W. M. Keck Foundation.

${ }^{7}$ Hubble Fellow.

${ }^{8}$ Visiting Astronomer at the Infrared Telescope Facility, which is operated by the University of Hawaii under contract NNH14CK55B with the National Aeronautics and Space Administration.

${ }^{9}$ Hubble Fellow.

${ }^{10}$ NSF Graduate Research Fellow.
}

of these scenarios are difficult with the relatively small sample of known objects (Bowler 2016).

In principle, clues about their origin can be inferred from a comparative abundance analysis with their host stars (e.g., Konopacky et al. 2013; Barman et al. 2015), measurements of their occurrence rate over time, long-term monitoring of their orbits (e.g., Ginski et al. 2014; Rameau et al. 2016; Blunt et al. 2017), the physical properties of their circumplanetary disks (Bowler et al. 2015; Kraus et al. 2015; Stamatellos \& Herczeg 2015), and constraints on their mass and semimajor axis distributions (Biller et al. 2013; Brandt et al. 2014; Reggiani et al. 2016). Indeed, Bryan et al. (2016) recently concluded that dynamical scattering is probably not the dominant origin of wide PMCs based on the lack of close-in scatterers, the low rate of close-in giant planets in the field, and early orbital constraints for wide PMCs.

Regardless of their formation route, the favorable angular separations and contrasts of wide PMCs make them attractive targets for detailed spectroscopic characterization in the nearinfrared (e.g., Bowler et al. 2014; Gauza et al. 2015). As such, they represent excellent targets to study the atmospheres of young gas giant planets and serve as empirical templates for discoveries with second-generation planet-finding instruments like the Gemini Planet Imager (GPI), Spectro-Polarimetric High-contrast Exoplanet REsearch (SPHERE), and Subaru Coronagraphic Extreme Adaptive Optics (SCExAO).

Kraus et al. (2014) presented the discovery of faint companions comoving with the pre-main sequence stars FW Tau AB, ROXs $42 \mathrm{~B}$, and ROXs 12 at projected separations between 100 and 
$400 \mathrm{au}$. At the young ages of these systems (1-10 Myr), the implied masses of the companions span about 5-20 $M_{\text {Jup }}$, assuming hot-start cooling models. Follow-up spectroscopy by Bowler et al. (2014) showed that ROXs 42B b is a young early-L dwarf with clear spectroscopic signs of low surface gravity. In contrast, the companion to FW Tau exhibits a mostly featureless near-infrared pseudocontinuum spectrum with strong veiling and emission lines indicating ongoing accretion and outflow activity. The implication is that the faint companion to FW Tau may be a brown dwarf with an edge-on disk rather than a widely separated planet, as suggested from photometry alone. Follow-up spectroscopic confirmation is evidently a critical step in confirming the low temperatures and masses of young planet candidates found with direct imaging.

Here we present near-infrared integral-field spectroscopy of the substellar companion to ROXs 12 (2MASS J16262803-2526477), an M0-type pre-main-sequence $\operatorname{star}^{11}$ near the boundary between the Ophiuchus and Upper Scorpius star-forming regions. ROXs $12 \mathrm{~B}^{12}$ was first identified as a candidate companion by Ratzka et al. (2005) at 1".78 (240 au in projected separation) and confirmed to be comoving with its host star by Kraus et al. (2014). More recently, Bryan et al. (2016) obtained follow-up high-contrast imaging of this system and found that ROXs $12 \mathrm{~B}$ has undergone measurable orbital motion based on astrometry spanning about $15 \mathrm{yr}$. As one of only a handful of young $(<10$ Myr) very-low-mass companions at wide orbital distances beyond $100 \mathrm{au}, \mathrm{ROXs} 12 \mathrm{~B}$ offers a valuable opportunity to study the atmosphere of a young object spanning the brown dwarf-planetary mass boundary.

In addition to characterizing the atmosphere of ROXs $12 \mathrm{~B}$, we also show that the young disk-bearing star 2MASS J16262774-2527247 (hereafter 2M1626-2527), located 37" south of ROXs 12, is likely a wide binary companion based on a consistent radial velocity and proper motion with ROXs 12 . Figure 1 shows an overview of the system. The combination of rotation periods from $K 2$ and projected rotational velocities from our high-resolution spectra allows us to assess the mutual inclinations of both stars. Combining these results with orbital inclination constraints for ROXs $12 \mathrm{~B}$ enables us to then examine whether the ROXs 12 system is in spin-orbit alignment, as expected for a planet forming in a disk. To

\footnotetext{
11 There has been some confusion regarding the coordinates of ROXs 12 in the literature. Montmerle et al. (1983) originally identified 47 "Rho Oph X-ray" (ROX) detections in observations with the Einstein Observatory X-ray telescope. ROX 12 is listed at $\alpha_{\mathrm{J} 2000.0}=16: 26: 24.8, \delta_{\mathrm{J} 2000.0}=-25: 27: 28$ with a $40^{\prime \prime}$ positional uncertainty. In a follow-up study to identify the sources of the X-ray emission, Bouvier \& Appenzeller (1992) listed ROXs 12 ("ROX star 12 "), the candidate optical counterpart to the ROX 12 detection, as the star at $\alpha_{\mathrm{J} 2000.0}=16: 26: 28.0, \quad \delta_{\mathrm{J} 2000.0}=-25: 26: 47$ (also known as 2MASS J16262803-2526477). Later, Ratzka et al. (2005) used speckle imaging to identify a candidate companion to ROXs 12 at a position angle (P.A.) of $10^{\circ} 3$ a separation of 1.775 , and a flux ratio of $\Delta K=5.7 \mathrm{mag}$, but the coordinates they listed are for a different star at $\alpha_{\mathrm{J} 2000.0}=16: 26: 27.75$, $\delta_{\mathrm{J} 2000.0}=-25: 27: 24.7$ (also known as 2MASS J16262774-2527247). Kraus et al. (2014) obtained follow-up adaptive-optics imaging of ROXs 12 (2MASS J16263803-2526477); while constructing the target list, they used coordinates based on the 2MASS source closest to the position in Bouvier \& Appenzeller (1992), observing the correct source. However, in the manuscript that confirmed comovement for the companion identified by Ratzka et al., they adopted the same (incorrect) coordinates for the star 37" south of ROXs 12 that were listed by Ratzka et al. Most of the observations we collected between 2011 and 2015 targeted the erroneous published coordinates for ROXs 12 but nevertheless provided useful data for the third companion 2MASS J16262774-2527247.

12 Here we adopt "B" rather than "b" as used in the discovery paper because the mass we infer for this companion is securely above the deuterium-burning limit.
}

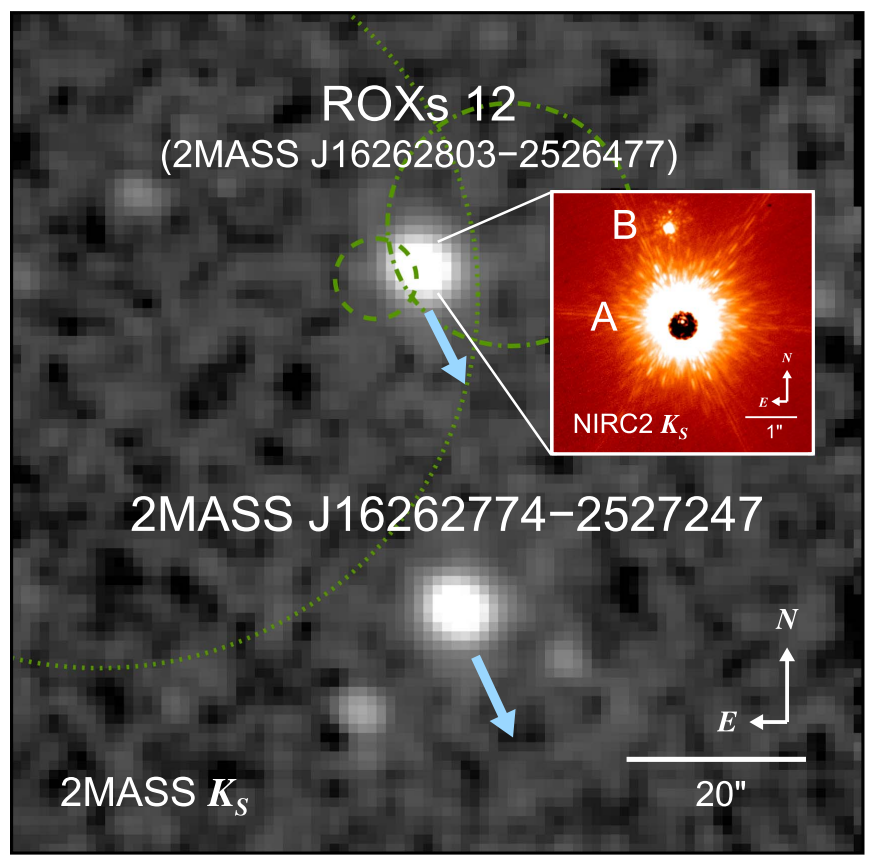

Figure 1. Overview of the ROXs 12 triple system. This seeing-limited 2MASS $K_{S}$-band image shows the wide binary ROXs 12 A (2MASS J16262803-2526477) and 2MASS J16262774-2527247 separated by 37" (5100 au). The substellar companion ROXs 12 B is located 1".8 (240 au) from its host star, seen here in the inset $K_{S}$-band adaptive-optics image from Keck/ NIRC2. Green circles show the X-ray error circles from the Einstein Observatory (dotted; Harris et al. 1996), ROSAT (dash-dotted; Voges et al. 2000), and Swift-XRT (dashed; Evans et al. 2014). Proper-motion vectors for ROXs 12 A and 2MASS J16262774-252724 are shown as blue arrows indicating the direction of motion (see Section 3.3); the length of the arrows has been magnified to make them visible. North is up, and east is to the left.

place ROXs 12 B in context, we conclude with an overview of accreting subdisks around young PMCs and derive the frequency of accreting circumplanetary disks from companions with existing moderate-resolution $J$-band spectra.

\section{Observations}

\subsection{Gemini-North/Near-infrared Integral Field Spectrometer $\mathrm{J}$-Band Spectroscopy of ROXs $12 \mathrm{~B}$}

We acquired moderate-resolution $(R \equiv \lambda / \delta \lambda \approx 6000)$ $J$-band spectroscopy of ROXs 12 B spanning $1.15-1.36 \mu \mathrm{m}$ with the Near-infrared Integral Field Spectrometer (NIFS; McGregor et al. 2003) at the Gemini-North $8.1 \mathrm{~m}$ telescope on UT 2016 April 18 and 22 (Gemini Program ID: GN-2016A-Q37). The facility adaptive optics (AO) system ALTAIR (Christou et al. 2010) provided diffraction-limited correction using the Gemini laser guide star system with ROXs 12 as a bright $(R=13.5 \mathrm{mag})$ tip-tilt reference. NIFS has a $3^{\prime \prime} \times 3^{\prime \prime}$ field of view with 0 ". $1 \times 0$ !' 04 rectangular spaxels. To better sample the point-spread function (PSF) wing of the host star, we rotated the instrument so that the short side of the spaxels was oriented in the direction of the ROXs 12 A and B P.A. with the host star located immediately off the detector (see Figure 2). Our observations of ROXs $12 \mathrm{~B}$ were then carried out in an ABBA pattern by nodding $1 . " 5$ orthogonal to the binary P.A.

We acquired a total of 40 minutes (eight exposures of $300 \mathrm{~s}$ each) with the $J$-band grating centered at $1.25 \mu \mathrm{m}$ and $\mathrm{ZJ}$ filter on UT 2016 April 18 in queue mode. On UT 2016 April 22, an additional 70 minutes (14 exposures of $300 \mathrm{~s}$ each) were taken 
Table 1

Spectroscopic Observations

\begin{tabular}{|c|c|c|c|c|c|c|c|c|}
\hline Object & $\begin{array}{l}\text { Date } \\
\text { (UT) }\end{array}$ & $\begin{array}{l}\text { Telescope/ } \\
\text { Instrument }\end{array}$ & Filter & $\begin{array}{l}\text { Slit Width } \\
\text { (arcsec) }\end{array}$ & $\begin{array}{l}\text { Plate Scale } \\
\left(\text { mas pixel }^{-1}\right)\end{array}$ & $\begin{array}{l}\text { Tot. Exp. } \\
\text { (minutes) }\end{array}$ & $\begin{array}{l}\text { Resolution } \\
(=\lambda / \delta \lambda)\end{array}$ & Standard $^{\mathrm{a}}$ \\
\hline ROXs $12 \mathrm{~B}$ & 2016 Apr $18+22$ & Gemini-North/NIFS & $J$ & $\cdots$ & $100 \times 40$ & 110 & 6000 & HD 145127 \\
\hline ROXs 12 B & 2016 May 22 & Keck/OSIRIS & $K b b$ & $\cdots$ & 50 & 50 & 3800 & HIP 69021 \\
\hline ROXs 12 B & 2016 May 22 & Keck/OSIRIS & $H b b$ & $\cdots$ & 50 & 60 & 3800 & HIP 93691 \\
\hline ROXs 12 B & 2016 May 22 & Keck/OSIRIS & $J b b$ & $\cdots$ & 50 & 90 & 3800 & HIP 93691 \\
\hline ROXs 12 A & 2011 Jun 28 & Keck/HIRES & KV418 & 0.861 & $\cdots$ & 5 & 48000 & $\cdots$ \\
\hline ROXs 12 A & 2016 Jul 26 & McDonald $2.7 \mathrm{~m} /$ IGRINS & $\cdots$ & 0.98 & $\cdots$ & 30 & 45000 & HD 155379 \\
\hline 2M1626-2527 & 2011 Apr 29 & IRTF/SpeX & $\ldots$ & 0.3 & $\cdots$ & 8 & 2000 & HD 144925 \\
\hline 2M1626-2527 & 2014 May 21 & Mayall/RC-Spec & $G G 495$ & 1.5 & $\cdots$ & 20 & 2600 & HZ 44 \\
\hline 2M1626-2527 & 2015 May 03 & Keck/HIRES & $G G 475$ & 0.861 & $\cdots$ & 10 & 48000 & $\ldots$ \\
\hline 2M1626-2527 & 2016 Jul 26 & McDonald $2.7 \mathrm{~m} /$ IGRINS & $\ldots$ & 0.98 & $\cdots$ & 30 & 45000 & HD 155379 \\
\hline
\end{tabular}

Note.

${ }^{\text {a }}$ Telluric or radial velocity standard.

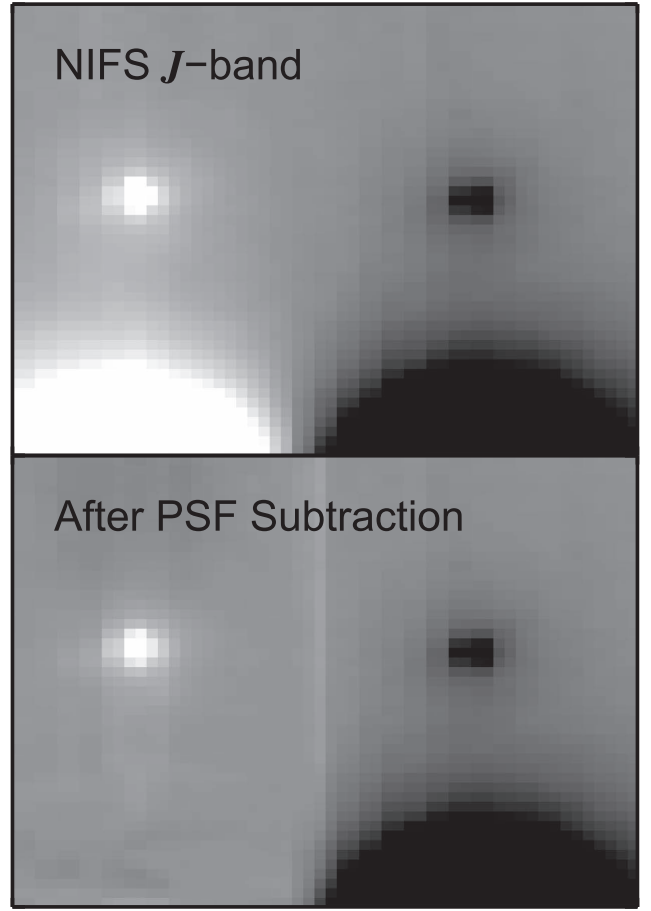

Figure 2. Gemini-North/NIFS collapsed image of ROXs 12 B before (top) and after (bottom) PSF subtraction of the host star using a Gaussian profile. The positive and negative images are caused by pairwise subtraction of two consecutive frames nodded by 1 ". 5 . ROXs $12 \mathrm{~A}$ is positioned outside the field of view, but uncorrected residual light is visible at the bottom of the array.

with the same configuration. On both nights, the A0V standard HD 145127 was targeted at a similar airmass for telluric correction. Our observations are summarized in Table 1.

Basic data reduction was carried out with NIFS reduction packages in IRAF that include flat-fielding, bad-pixel interpolation, sky subtraction, and image rectification to data cubes. ROXs $12 \mathrm{~B}$ is clearly visible in each cube but overlaps with the PSF wing from the host star. To remove this low-level contaminating flux, we performed PSF subtraction in the same fashion as in Bowler et al. (2014) for NIFS data of ROXs 42B b. For each column of each spectral channel, we masked out the companion and then fit and subtracted various parameterized PSF models to the 2D wing of ROXs 12 A using the Levenberg-Marquardt least-squares curve-fitting package MPFIT (Markwardt 2009). Gaussian, Lorentzian, and Moffat
PSF models were tested to examine their influence on the extracted spectra. All three profiles resulted in similar spectra; ultimately, we adopted the Gaussian model, as this produced the lowest systematic oversubtraction or undersubtraction in the region surrounding ROXs 12 B (Figure 2). After PSF subtraction, we extracted the spectra from each cube using aperture photometry and median-combined them after scaling individual spectra to their median values. Telluric correction was performed using the xtellcor_general routine in the Spextool data reduction package for the InfraRed Telescope Facility (IRTF) SpeX instrument (Vacca et al. 2003; Cushing et al. 2004). These steps were carried out separately for each night, and the resulting spectra were subsequently combined by calculating the weighted mean and uncertainty of both spectra. Note that standards were taken immediately before and after the science observations, and the signal-to-noise ratio $(\mathrm{S} / \mathrm{N})$ levels were low for individual spectra, so we performed telluric correction on the coadded spectra from each night instead of carrying this out for each separate exposure. The final NIFS spectrum has an effective on-source integration time of 110 minutes and is shown in Figure 3 after having been dereddened by $A_{V}=1.8 \mathrm{mag}$ - the extinction to ROXs $12 \mathrm{~A}$ measured by Rizzuto et al. (2015) based on moderate-resolution optical spectroscopy-following the extinction curve from Fitzpatrick (1985).

\subsection{Keck/OSIRIS Near-infrared Spectroscopy of ROXs $12 \mathrm{~B}$}

We targeted ROXs $12 \mathrm{~B}$ with the OH-Suppressing InfraRed Imaging Spectrograph (OSIRIS; Larkin et al. 2006; Mieda et al. 2014) using natural guide star adaptive optics (NSG AO; Wizinowich 2013) at the Keck I telescope on 2016 May 22 UT. Broadband filters were used with the 50 mas spaxel ${ }^{-1}$ plate scale, producing a $16 \times 64$ spaxel $(0$ "! $8 \times 3$ "! 2$)$ rectangular field of view with a spectral resolution of $R \approx 3800$. The sky was clear with seeing between 0.15 and $0 . " 8$ throughout the night. To minimize contamination from the host star, we oriented the long axis of the array to be orthogonal to the binary P.A. Our observations consisted of nodded ABBA sequences with about $1^{\prime \prime}$ offsets for pairwise sky subtraction. A total of 50 minutes of integration time was acquired in the $K b b$ band (10 $300 \mathrm{~s}$ exposures), 60 minutes in the $H b b$ band (12 $300 \mathrm{~s}$ exposures), and 90 minutes in the $J$ band (18 $300 \mathrm{~s}$ exposures). Multiple A0V standards were targeted between filter changes at an airmass similar to that of the science observations (see Table 1). 


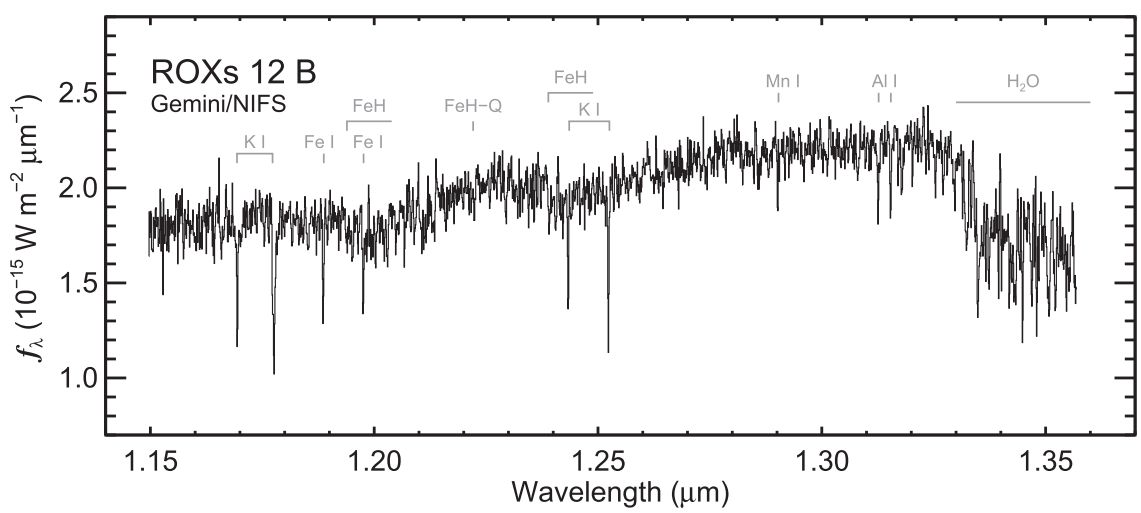

Figure 3. Gemini-North/NIFS $1.15-1.36 \mu \mathrm{m}$ spectrum of ROXs $12 \mathrm{~B}$. Strong atomic and molecular species are labeled in gray, including K I, Fe I, Mn I, and Al I. The resolving power is $\approx 6000$, and the spectrum has been corrected for reddening $\left(A_{V}=1.8 \mathrm{mag}\right)$.

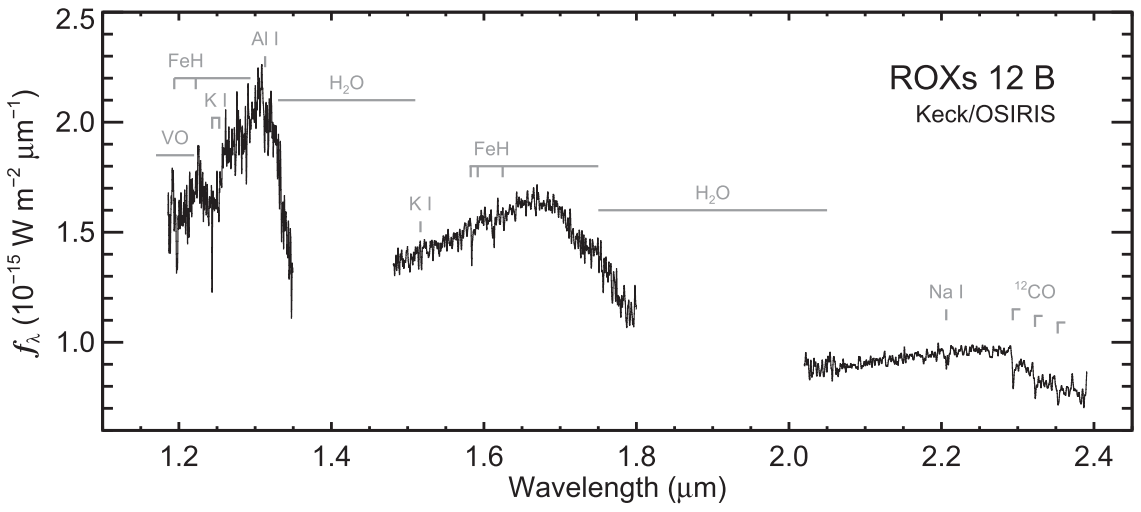

Figure 4. Keck/OSIRIS $1.18-2.38 \mu \mathrm{m}$ spectrum of ROXs 12 B. Major atomic and molecular species are labeled in gray. Individual bandpasses have been fluxcalibrated to the photometry listed in Table 2, Gaussian-smoothed from a native resolving power of $R=3800$ to $R=1000$ to improve the $\mathrm{S} / \mathrm{N}$ for visual purposes, and dereddened by $A_{V}=1.8 \mathrm{mag}$.

Basic data reduction was carried out using the OSIRIS data reduction pipeline. Images were flat-fielded, corrected for bad pixels, and assembled into data cubes using the latest rectification matrices provided by Keck Observatory. We then extracted the spectra from the data cubes using aperture photometry at each wavelength at the centroided location of the target in median-collapsed cubes. Spectra were mediancombined after scaling them to their individual median levels. Telluric correction was then carried out with the xtellcor general routine in Spextool (Vacca et al. 2003; Cushing et al. 2004). Each spectral band was then flux-calibrated using the photometry of ROXs 12 B (Figure 4), which was derived by transforming the 2MASS photometry of ROXs $12 \mathrm{~A}$ to the MKO system using relations from Leggett et al. (2006) and then using the relative photometry of ROXs $12 \mathrm{AB}$ from Kraus et al. (2014). The resulting photometry is reported in Table 2.

\subsection{Harlan J. Smith Telescope/IGRINS High-resolution Near-infrared Spectroscopy}

We obtained high-resolution $(R=45000) \quad 1.45-2.45 \mu \mathrm{m}$ spectra of ROXs 12 A and 2M1626-2527 on UT 2016 July 26 with the Immersion GRating INfrared Spectrometer (IGRINS; Park et al. 2014; Mace et al. 2016) at the McDonald Observatory's $2.7 \mathrm{~m}$ Harlan J. Smith Telescope to measure radial velocities for both components of this common proper-motion pair in order to test whether they share common space velocities. Six exposures of $300 \mathrm{~s}$ each were acquired while nodding in an ABBA pattern along the slit, totaling 30 minutes of integration for each target.
The A0V star HD 155379 was observed on the same night. Spectra were extracted and reduced using version 2.1 of the IGRINS reduction pipeline ${ }^{13}$ following the description in Bowler et al. (2017). The $S / N$ per pixel of both spectra ranges from 80 to 100. In summary, after bias subtraction and flat-fielding, the spectra were optimally extracted and cross-correlated with over 100 other IGRINS spectra of comparable spectral type taken over the past $2 \mathrm{yr}$ to measure radial velocities. The final radial velocities for ROXs $12 \mathrm{~A}$ and 2M1626-2527 are $-6.09 \pm 0.16$ and $-6.03 \pm 0.16 \mathrm{~km} \mathrm{~s}^{-1}$, respectively, where the uncertainties are dominated by the transformation to the external reference frame (as opposed to relative uncertainties, which are $0.04 \mathrm{~km} \mathrm{~s}^{-1}$ ).

\subsection{Mayall/Ritchey-Chretien Spectrograph Optical Spectroscopy of 2M1626-2527}

We observed 2M1626-2527 with the Ritchey-Chretien Spectrograph (RC-Spec) using the T2KA CCD at the Kitt Peak National Observatory's 4 m Mayall telescope on UT 2014 May 21. The $1 . ! 5 \times 98^{\prime \prime}$ slit was used with the BL420 grating, resulting in a resolving power of $R \approx 2600$ spanning 6300-9200 $\AA$. The slit was oriented in a fixed north-south direction throughout the night. We targeted 2M1626-2527 near transit at an airmass of 1.86 to minimize effects from differential atmospheric refraction. However, because the slit was not oriented exactly at the parallactic angle, some slit losses may have occurred that may have affected the slope of

\footnotetext{
${ }^{13}$ https://github.com/igrins/plp
} 
Table 2

Physical, Kinematic, and Photometric Properties of the ROXs 12 Triple System

\begin{tabular}{|c|c|c|c|c|}
\hline Property & ROXs 12 A & ROXs $12 \mathrm{~B}$ & $2 \mathrm{M} 1626-2527$ & Reference \\
\hline 2MASS ID & $\mathrm{J} 16262803-2526477$ & $\cdots$ & J16262774-2527247 & (1) \\
\hline$B$ (mag) & $16.172 \pm 0.141$ & $\cdots$ & $17.1 \pm 0.7$ & (2) \\
\hline$V$ (mag) & $14.346 \pm 0.066$ & $\ldots$ & $15.8 \pm 0.3$ & (2) \\
\hline$R$ (mag) & 13.5 & $\ldots$ & 15.3 & (3) \\
\hline$r^{\prime}$ (mag) & 13.5 & $\ldots$ & 15.8 & (4) \\
\hline$I(\mathrm{mag})$ & $11.870 \pm 0.04$ & $\ldots$ & $12.91 \pm 03$ & (5) \\
\hline$J_{2 \mathrm{MASS}}(\mathrm{mag})$ & $10.282 \pm 0.024$ & $15.82 \pm 0.03^{\mathrm{a}}$ & $11.021 \pm 0.024$ & $(1),(6)$ \\
\hline$H_{2 \text { MASS }}(\mathrm{mag})$ & $9.386 \pm 0.026$ & $14.83 \pm 0.03^{\mathrm{a}}$ & $9.930 \pm 0.026$ & $(1),(6)$ \\
\hline$K_{s, 2 \text { MASS }}(\mathrm{mag})$ & $9.099 \pm 0.025$ & $14.14 \pm 0.03^{\mathrm{a}}$ & $9.211 \pm 0.025$ & $(1),(6)$ \\
\hline$L^{\prime}(\mathrm{mag})$ & {$[9.1 \pm 0.1]^{\mathrm{b}}$} & $13.2 \pm 0.1$ & {$[9.2 \pm 0.1]^{\mathrm{b}}$} & (6) \\
\hline$W 1$ (mag) & $8.805 \pm 0.023$ & $\ldots$ & $8.360 \pm 0.023$ & (7) \\
\hline$W 2$ (mag) & $8.712 \pm 0.021$ & $\ldots$ & $7.831 \pm 0.021$ & (7) \\
\hline W3 (mag) & $8.393 \pm 0.042$ & $\ldots$ & $5.983 \pm 0.017$ & (7) \\
\hline$W 4$ (mag) & $6.558 \pm 0.089$ & $\ldots$ & $3.784 \pm 0.025$ & (7) \\
\hline$\mu_{\alpha} \cos \delta\left(\operatorname{mas~yr}^{-1}\right)$ & $-12.7 \pm 2.3$ & $\ldots$ & $-11.9 \pm 2.3$ & (8) \\
\hline$\mu_{\delta}\left(\operatorname{mas} \mathrm{yr}^{-1}\right)$ & $-29.0 \pm 2.3$ & $\ldots$ & $-30.5 \pm 2.3$ & (8) \\
\hline$v_{\mathrm{rad}}\left(\mathrm{km} \mathrm{s}^{-1}\right)^{\mathrm{c}}$ & $-6.09 \pm 0.16$ & $\ldots$ & $-6.03 \pm 0.16$ & (9) \\
\hline$v_{\text {rad }}\left(\mathrm{km} \mathrm{s}^{-1}\right)^{\mathrm{d}}$ & $-5.67 \pm 0.12$ & $\ldots$ & $-6.97 \pm 0.15$ & (9) \\
\hline$v \sin i\left(\mathrm{~km} \mathrm{~s}^{-1}\right)^{\mathrm{d}}$ & $8.2 \pm 0.7$ & $\ldots$ & $4.5 \pm 1.0$ & (9) \\
\hline $\log \left(L_{\mathrm{bol}} / L_{\odot}\right)^{\mathrm{e}}$ & $-0.57 \pm 0.06$ & $-2.87 \pm 0.06$ & $-0.81 \pm 0.06$ & (9) \\
\hline $\operatorname{Mass}\left(M_{\odot}\right)$ & $0.65_{-0.09}^{+0.05}$ & $0.0167 \pm 0.0014$ & $0.5_{-0.1}^{+0.1}$ & (9) \\
\hline$T_{\text {eff }}(\mathrm{K})$ & $3900 \pm 100$ & $3100_{-500}^{+400}$ & $3700 \pm 150$ & (9) \\
\hline$P_{\text {rot }}(\mathrm{d})$ & $9.1 \pm 0.4$ & $\ldots$ & $3.30 \pm 0.05$ & (9) \\
\hline Age (Myr) & $6_{-2}^{+4}$ & $6_{-2}^{+4}$ & $8_{-4}^{+7} \mathrm{Myr}$ & (9) \\
\hline Spectral type & $\mathrm{M} 0 \pm 0.5$ & $\mathrm{~L} 0 \pm 2$ & $\mathrm{M} 1 \pm 1$ & (9), (10) \\
\hline$i_{*}(\operatorname{deg})^{\mathrm{f}}$ & $77_{-9}^{+7}$ & $\ldots$ & $17_{-4}^{+5}$ & (9) \\
\hline$R_{*}\left(R_{\odot}\right)^{\mathrm{g}}$ & $1.14 \pm 0.07$ & $\ldots$ & $0.96 \pm 0.08$ & (9) \\
\hline
\end{tabular}

Notes.

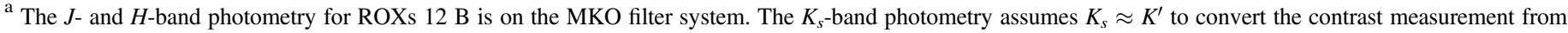
Kraus et al. (2014) to an apparent magnitude.

b Estimated $L^{\prime}$-band magnitudes assuming $K-L^{\prime}=0.0 \pm 0.1 \mathrm{mag}$ for M0 and M1 spectral types (Golimowski et al. 2004).

${ }^{\mathrm{c}}$ From our IGRINS high-resolution near-infrared spectra.

${ }^{\mathrm{d}}$ From our HIRES high-resolution optical spectra.

e Assumes a distance of $137 \pm 10 \mathrm{pc}$.

${ }^{f}$ Line-of-sight stellar inclination.

g Radius from bolometric luminosity and effective temperature.

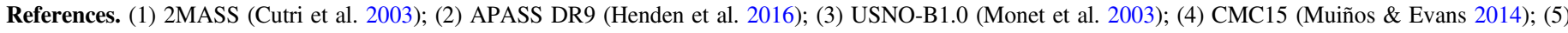

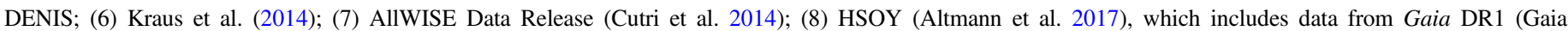
Collaboration et al. 2016); (9) this work; (10) Rizzuto et al. (2015).

our RC-Spec spectrum. These data are therefore useful for spectral classification but not for detailed reddening measurements. A single exposure was acquired with the GG495 filter and a total integration time of $1200 \mathrm{~s}$.

The raw data was first bias-subtracted and flat-fielded to remove pixel-to-pixel sensitivity variations. Night sky lines were removed using median sky values in the spatial direction on either side of the spectrum. We then extracted the spectrum of the science target by summing flux in the spatial direction. The overall throughput response was then corrected using the spectrophotometric standard HZ 44. Finally, wavelength calibration was achieved using HeNeAr lamp observations taken throughout the night. The optical spectrum of 2M1626-2527 is discussed in more detail in Section 3.4.

\subsection{IRTF/SpeX Moderate-resolution Near-infrared Spectroscopy of 2M1626-2527}

2M1626-2527 was targeted with the IRTF's SpeX instrument (Rayner et al. 2003) in short cross-dispersed mode on UT 2011
April 29. We used the 0.33 slit rotated to the parallactic angle, which yielded a mean resolving power of $\approx 2000$ across the $0.8-2.4 \mu \mathrm{m}$ spectrum. Four nodded pairs were obtained with $60 \mathrm{~s}$ per exposure in an ABBA pattern. The A0V standard HD 144925 was observed prior to our science target. Standard data reduction, including spectral extraction, wavelength calibration, and telluric correction, was carried out using the Spextool package (Vacca et al. 2003; Cushing et al. 2004). The near-infrared spectrum of 2M1626-2527 is discussed in more detail in Section 3.4.

\subsection{Keck/HIRES High-resolution Optical Spectroscopy}

We obtained high-resolution $(R \approx 48,000)$ optical spectra of ROXs 12 A and 2M1626-2527 with the High Resolution Echelle Spectrometer (HIRES; Vogt et al. 1994) at Keck Observatory on UT 2011 June 28 and UT 2015 May 3, respectively. Both spectra were optimized for long wavelengths with the red cross-disperser. A single $300 \mathrm{~s}$ exposure of ROXs 12 A was taken with the $\mathrm{C} 1$ decker using the KV418 orderblocking filter, resulting in a slit size of 7 !" $0 \times 0$ "! 861 
on the sky and a wavelength range of $4310-8770 \AA$. For 2M1626-2527, we obtained one $600 \mathrm{~s}$ exposure spanning a wavelength range of $4800-9220 \AA$ using the $\mathrm{C} 1$ decker, the GG475 order-blocking filter, and a slit size of 7 !" $0 \times 0$ "! 861 .

Basic data reduction and spectral extraction were carried out following the description in Kraus et al. (2011). The extraction pipeline MAKEE was used for bias subtraction, flat-fielding, cosmic-ray rejection, spectral extraction, and wavelength calibration. Zero-point corrections to the wavelength solution were derived by cross-correlating the telluric band of the O7V star S Mon. Radial velocities and projected rotational velocities $\left(v \sin i_{*}\right)$ were then measured for both targets using early-M dwarf radial velocity (RV) standards from Chubak et al. (2012) after removing orders with strong telluric features or low $\mathrm{S} / \mathrm{N}$. A broadening function (Rucinski 1999) was measured relative to these standards to find the absolute RV and line broadening, which was then translated into projected rotational velocities using rotationally broadened template spectra to empirically correlate line broadening and projected rotational velocities following Kraus et al. (2017). Our final RV values from HIRES are $-5.67 \pm 0.12 \mathrm{~km}^{-1}$ for ROXs 12 and $-6.97 \pm 0.15 \mathrm{~km} \mathrm{~s}^{-1}$ for $2 \mathrm{M} 1626-2527$, comparable to the radial velocities we found from our IGIRNS spectra. The slight differences $\left(<1 \mathrm{~km} \mathrm{~s}^{-1}\right)$ between the HIRES and IGRINS RVs are likely caused by jitter in these young and active stars. Our $v \sin i_{*}$ measurements from this analysis are $8.2 \pm 0.7$ and $4.5 \pm 1.0 \mathrm{~km} \mathrm{~s}^{-1}$ for ROXs 12 and $2 \mathrm{M} 1626-2527$, respectively.

\subsection{K2 Time-series Photometry}

ROXs 12 (EPIC 203640875) and 2MASS J16262774-2527247 (EPIC 203637940) were both observed with $K 2$ (Howell et al. 2014), the extended mission of the Kepler spacecraft, during Campaign 2 between 2014 August 23 and November 10 (GO 2052, PI: K. Covey; GO 2063, PI: A. Kraus). The raw long-cadence (30 minutes) photometry for ROXs 12 was corrected for systematic features caused by pixel-to-pixel drift and spacecraft thruster firings using the reduction pipeline described in Vanderburg \& Johnson (2014). For 2MASS J16262774-2527247, we used the raw light curve instead of applying corrections, because this star exhibits very large photometric variations - up to $\sim 50 \%$ in amplitude-that are far larger than the systematics present in the data. Light curves for both targets are presented in Section 3.5.

\subsection{Keck/NIRC2 Aperture-masking Interferometry}

ROXs 12 was observed on UT 2011 April 24 using NIRC2's nine-hole aperture mask together with NSG AO at Keck Observatory as part of the multiplicity survey of Ophiuchus members by Cheetham et al. (2015). The observations consisted of four $20 \mathrm{~s}$ images ( $5 \mathrm{~s} \times 4$ coadds) in the $K p$ filter using NIRC2's narrow camera mode. After basic reduction (bias subtraction, flatfielding, and bad-pixel correction), we reexamined the images and found that the interferogram from ROXs $12 \mathrm{~B}$ is clearly visible in all four frames. This serendipitous detection opens the opportunity to search for a potential binary companion to ROXS $12 \mathrm{~B}$ at the Keck diffraction limit—analogous to brown dwarf-brown dwarf binary companions to stars like HD 130948 BC (Potter et al. 2002) and $\epsilon$ Indi Bab (Scholz et al. 2003; McCaughrean et al. 2004) - albeit at modest flux ratios because of flux loss inherent to this technique and ROXs $12 \mathrm{~B}$ 's intrinsic faintness.

Figure 5 displays the coadded image of the ROXs $12 \mathrm{AB}$ system after applying the distortion solution and north orientation
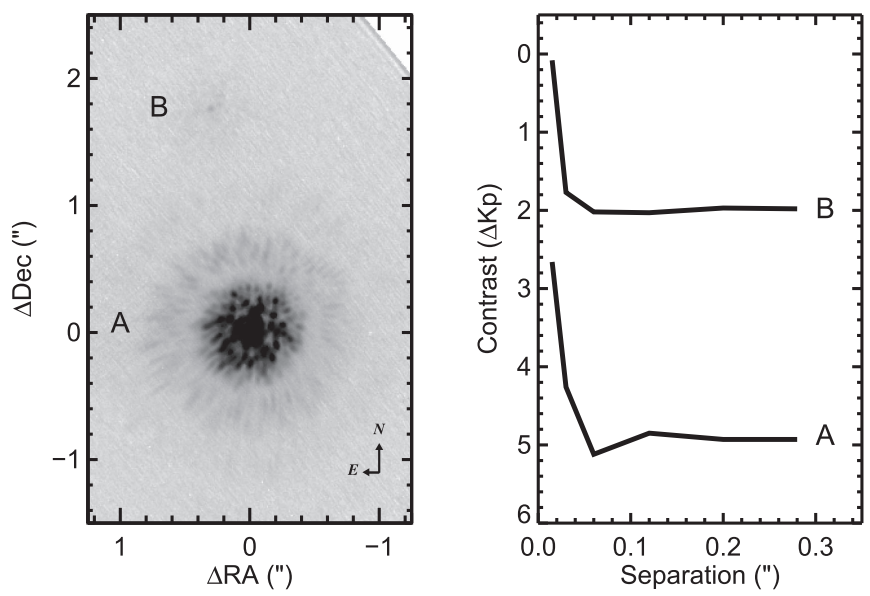

Figure 5. Adaptive-optics aperture-masking interferometry of ROXs $12 \mathrm{~A}$ and $B$ with the NIRC2 nine-hole mask at Keck Observatory. Left: The interferograms of ROXs $12 \mathrm{~A}$ and B in this $K p$-band image offer a way to search for additional companions below the telescope diffraction limit. Right: 99\% contrast curves for ROXs 12 B (this work) and the host star (see Cheetham et al. 2015).

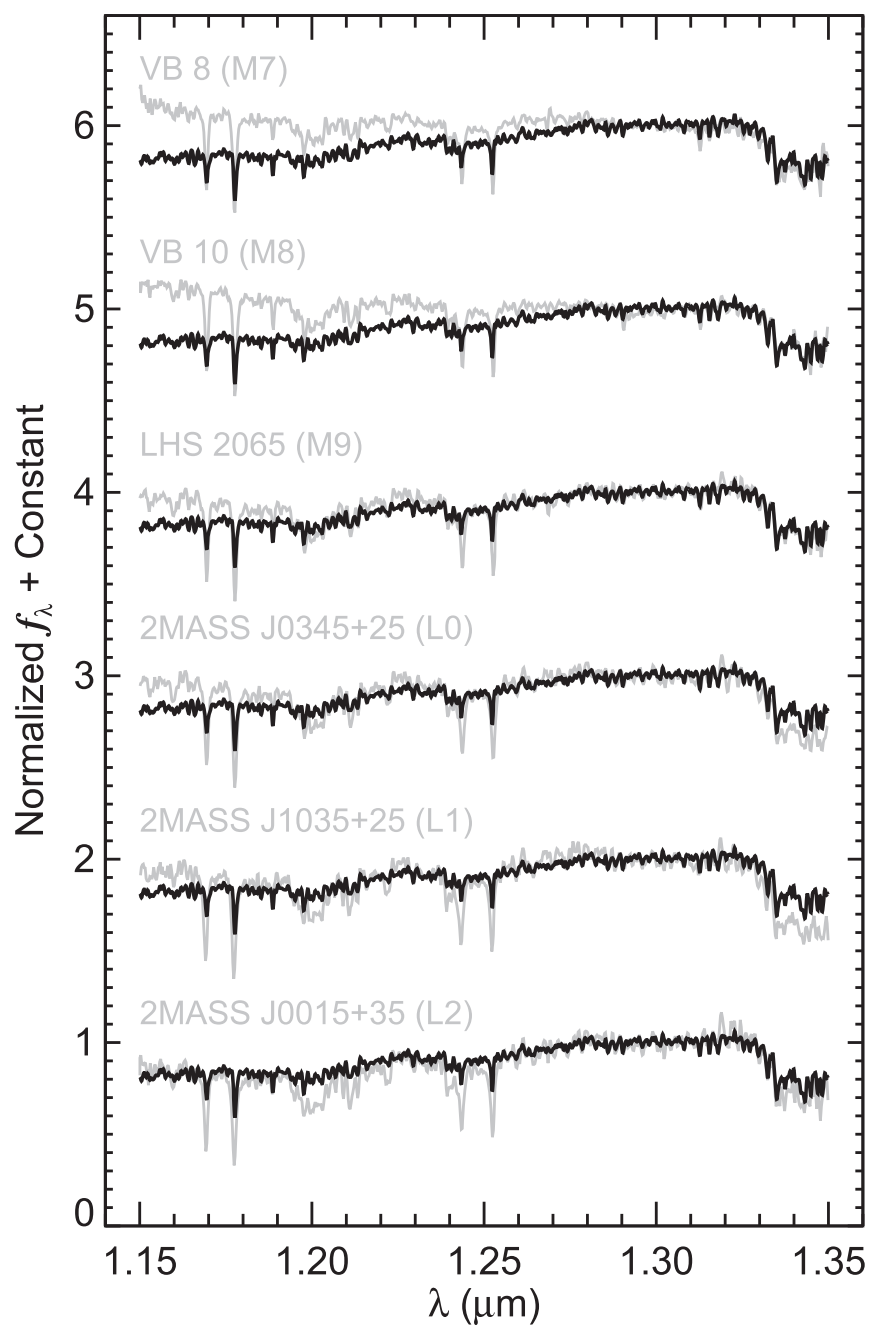

Figure 6. Comparison of our NIFS $J$-band spectrum of ROXs 12 B (black) to M7-L2 objects from BDSS (gray; McLean et al. 2003; McGovern et al. 2004). Our spectrum has been Gaussian-smoothed to match the resolving power of the BDSS spectra $(R \approx 2000)$ and dereddened by $A_{V}=1.8$ mag to account for extinction. 

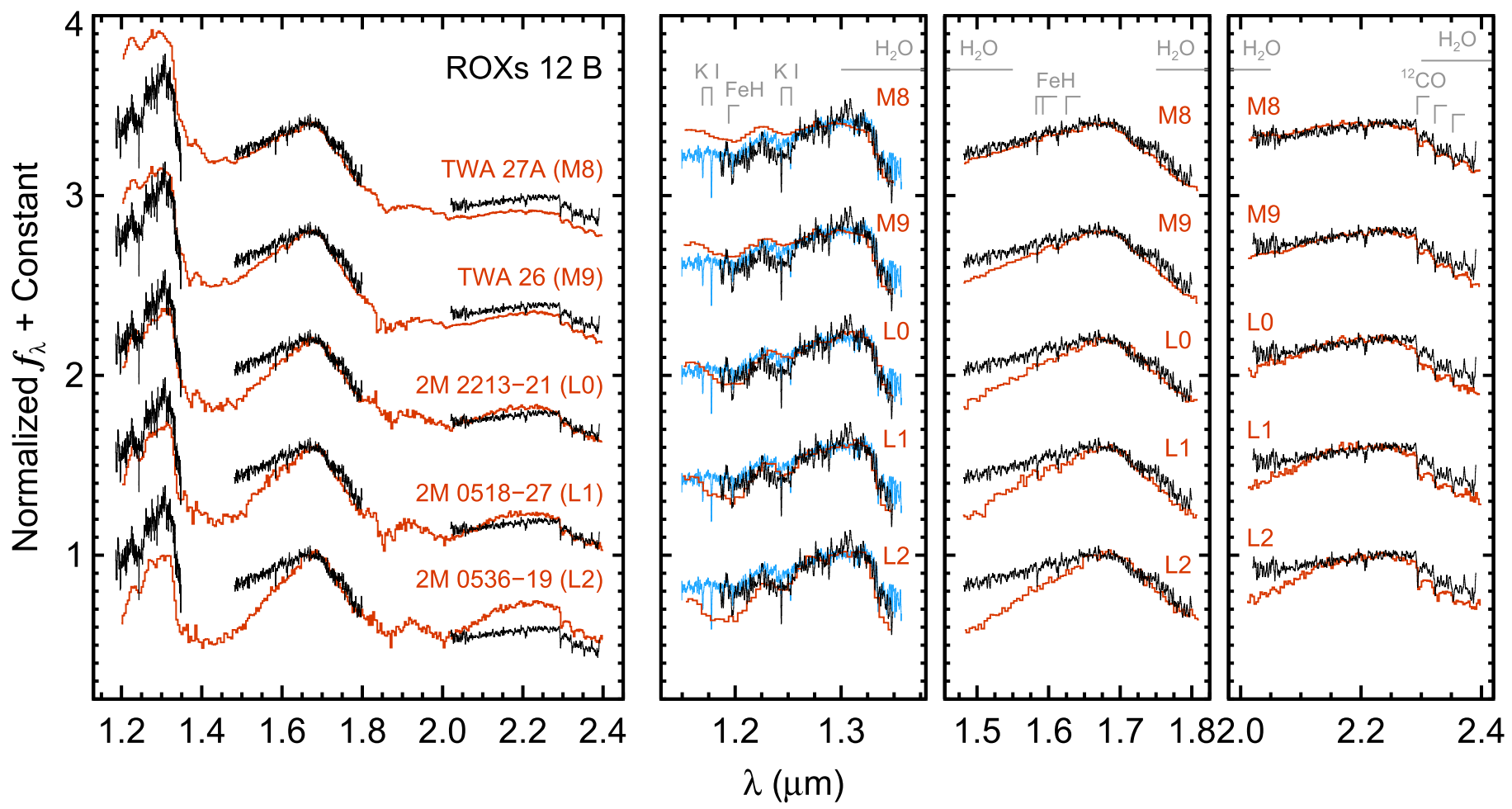

Figure 7. OSIRIS (black) and NIFS (blue) spectra of ROXs 12 B compared to VL-G standards from Allers \& Liu (2013). ROXs 12 B shows conflicting properties: the 1.2-2.4 $\mu \mathrm{m}$ most resembles the L0 template, but the individual bandpasses are closer to L1-L2 in the $J$ band and M8-M9 for the $H$ and $K$ bands. In particular, the blue side of the $H$-band slope is shallower than typical L dwarfs. We adopt L $0 \pm 2$ because of these discrepancies. The OSIRIS and NIFS spectra have been dereddened by $A_{V}=1.8$ mag to account for extinction.

from Yelda et al. (2010). Using the aperture-masking pipeline described in Kraus et al. (2008), we did not find evidence for an additional companion to ROXs $12 \mathrm{~B}$ down to angular scales of about 30 mas. The corresponding $99 \%$-level contrast curve in the $K p$ band over which we can exclude companions is $\{0.08,1.77$, $2.02,2.03,1.97,1.98\} \mathrm{mag}$ at $\{15,30,60,120,200,280\}$ mas. This corresponds to mass limits of $\approx 10 M_{\text {Jup }}$ at about 0 ". 1 based on the evolutionary models of Baraffe et al. (2015) for an age of $\approx 6 \mathrm{Myr}$ (see Section 3.6). It therefore appears that ROXs $12 \mathrm{~B}$ is single down to physical separations of $\approx 4 \mathrm{au}$, which is well within its Hill radius of about $50 \mathrm{au}$. Contrast limits for the host star from these same data are about 3 mag deeper; these are shown in Figure 5 and presented in Cheetham et al. (2015).

\section{Results}

\subsection{Spectral Properties of ROXs $12 \mathrm{~B}$}

Our moderate-resolution spectra of ROXs 12 B from Gemini-North/NIFS ( $J$ band) and Keck/OSIRIS $(J, H$, and $K$ bands) are shown in Figures 3 and 4 . The NIFS spectrum shows prominent atomic and molecular absorption features from $\mathrm{KI}, \mathrm{Fe}, \mathrm{MnI}, \mathrm{Al} \mathrm{I}, \mathrm{H}_{2} \mathrm{O}$, and $\mathrm{FeH}$, as well as hints of broad VO absorption at $\approx 1.2 \mu \mathrm{m}$. Notably absent is $\mathrm{Pa} \beta$ emission at $1.282 \mu \mathrm{m}$, a signature of active accretion from a circumsubstellar disk; such emission has been detected in several other young brown dwarf companions over the past few years (e.g., Seifahrt et al. 2007; Bowler et al. 2011).

We compare our NIFS spectrum to brown dwarfs from the Brown Dwarf Spectroscopic Survey (BDSS; McLean et al. 2003) in Figure 6. Relative to field objects, ROXs 12 B exhibits shallower K I doublets at $1.169 / 1.178 \mu \mathrm{m}$ and $1.244 /$ $1.253 \mu \mathrm{m}$, as well as diminished $\mathrm{FeH}$ molecular absorption
Table 3

ROXs 12 B NIFS $J$-Band Equivalent Widths

\begin{tabular}{|c|c|c|}
\hline $\begin{array}{l}\lambda_{0}{ }^{\mathrm{a}} \\
(\mu \mathrm{m})\end{array}$ & $\begin{array}{l}\text { Line } \\
\text { ID }\end{array}$ & $\begin{array}{l}\text { EW } \\
(\AA)\end{array}$ \\
\hline 1.1693 & K I & $1.10 \pm 0.09$ \\
\hline 1.1773 & K I & $2.60 \pm 0.10$ \\
\hline 1.1887 & $\mathrm{Fe} \mathrm{I}$ & $0.89 \pm 0.04$ \\
\hline 1.1976 & Fe I & $0.73 \pm 0.05$ \\
\hline 1.2436 & $\mathrm{~K}_{\mathrm{I}}$ & $0.88 \pm 0.04$ \\
\hline 1.2526 & $\mathrm{~K}_{\mathrm{I}}$ & $1.26 \pm 0.05$ \\
\hline 1.2903 & Mn I & $0.42 \pm 0.12$ \\
\hline 1.3127 & $\mathrm{Al} \mathrm{I}$ & $0.54 \pm 0.06$ \\
\hline 1.3154 & $\mathrm{Al} \mathrm{I}$ & $0.47 \pm 0.03$ \\
\hline
\end{tabular}

Note.

${ }^{\text {a }}$ Nominal air wavelength.

features at $\approx 1.195-1.205$ and $1.239 \mu \mathrm{m}$-all signs of low surface gravity (e.g., Gorlova et al. 2003; McGovern et al. 2004; Slesnick et al. 2004; Allers \& Liu 2013). The pseudocontinuum is a good match to the $\mathrm{L} 2$ template spectrum. Equivalent widths of absorption lines are reported in Table 3.

Our OSIRIS spectrum broadly resembles a late-M and early$\mathrm{L}$ dwarf with deep $\mathrm{H}_{2} \mathrm{O}$ bands at $\approx 1.4$ and $\approx 1.9 \mu \mathrm{m}$, FeH, ${ }^{12} \mathrm{CO}$, NaI, and weak KI. The triangular $H$-band shape is typical of low-gravity brown dwarfs and giant planets (e.g., Lucas et al. 2001; Kirkpatrick et al. 2006; Allers et al. 2007), offering independent evidence that the system is young; although, note that the triangular $H$-band shape does not strictly track low surface gravity (Allers \& Liu 2013).

In Figure 7, we compare our OSIRIS and NIFS spectra to very-low-gravity (VL-G) templates from Allers \& Liu (2013). 


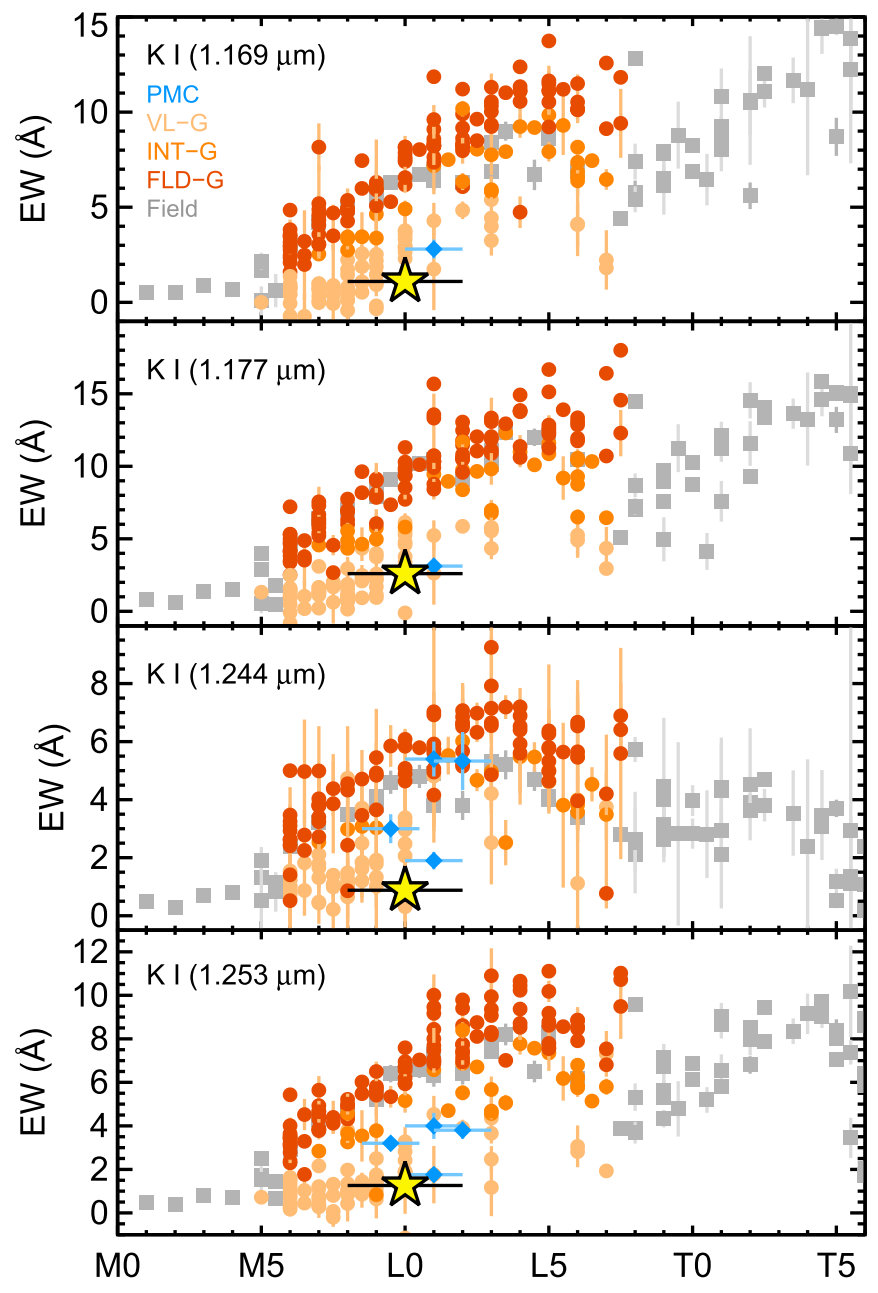

Figure 8. Equivalent widths of gravity-sensitive potassium features as a function of spectral type. Field objects without formal gravity classifications (gray squares) are from Cushing et al. (2005) and Martin et al. (2017); data for VL-G, intermediate gravity (INT-G), and field gravity (FLD-G) are from Allers \& Liu (2013) and Martin et al. (2017); and young PMCs are from Table 4. The yellow star denotes the position of ROXs $12 \mathrm{~B}$.

The full $1.2-2.4 \mu \mathrm{m}$ spectrum is most similar to the young L0 template, but each bandpass individually shows inconsistent matches: the $J$ band resembles L1-L2 objects, the $H$ band is closest to the M8 template, and the $K$ band appears similar to M8-M9 objects. Altogether, we adopt a near-infrared spectral type of $\mathrm{LO} \pm 2$ for ROXs $12 \mathrm{~B}$. Note that the OSIRIS broadband filter bandpasses do not cover the spectral regions used in the Allers \& Liu (2013) classification system, so we adopt visual-based classifications for ROXs $12 \mathrm{~B}$.

Equivalent widths of the $J$-band potassium doublets from our NIFS spectrum are shown relative to ultracool objects in Figure 8. High-gravity field objects possess deep lines that trace out an upper envelope of equivalent widths, whereas young objects exhibit weaker alkali features as a result of their low-pressure atmospheres. Compared to low-mass stars and brown dwarfs spanning a range of ages and surface gravities, ROXs 12 B has shallow potassium lines similar to those of VLG objects from Allers \& Liu (2013) and Martin et al. (2017), as well as young $(\lesssim 10 \mathrm{Myr})$ companions near and below the deuterium-burning limit, as measured from published spectra (Table 4).

\subsection{Physical Properties of ROXs $12 \mathrm{~B}$}

We implement two methods to assess the effective temperature and surface gravity of ROXs 12 B using atmospheric models. The first approach is based on maximum-likelihood fitting of the BT-Settl models (CIFIST2011bc version; Allard et al. 2011) following the general description in Bowler et al. (2009). In summary, the grid of models are first Gaussian-smoothed to the resolving power of the OSIRIS spectrograph $(R \approx 3800)$ and resampled onto the same wavelength grid as the data. A reduced $\chi^{2}$ value $\left(\chi_{\nu}^{2}\right)$ is calculated for each synthetic spectrum in the grid spanning $T_{\text {eff }}=$ $1100-4000 \mathrm{~K}\left(\Delta T_{\text {eff }}=100 \mathrm{~K}\right)$ and $\log g=2.5-5.5 \mathrm{dex}$ [cgs units throughout this work] $(\Delta \log g=0.5 \mathrm{dex})$. Similarly, a corresponding radius is derived for each model by making use of the factor that scales the emergent model spectrum to the observed flux-calibrated spectrum, as this quantity is also equal to the ratio of the object's radius to its distance squared. Here we adopt a distance of $137 \pm 10 \mathrm{pc}$, where the mean value reflects the distance measured to the dark cloud Lynds 1688 by Ortiz-León et al. (2017) and the uncertainty reflects the uncertainty of the membership of this system in the Ophiuchus versus Upper Sco star-forming regions (see Section 4.1).

In addition to fitting the entire 1.2-2.4 $\mu \mathrm{m}$ spectrum, we also fit the individual $J, H$, and $K$ bands using this method. The results are shown in Figure 9. The models qualitatively match the data quite well but produce different quantitative results depending on the bandpass, with effective temperatures ranging from 1600 to $2800 \mathrm{~K}$. The bottom panel of the figure shows the entire 1.2-2.4 $\mu \mathrm{m}$ spectrum; the best model does a poor job of reproducing the data by underestimating the flux at shorter wavelengths and overpredicting it in the $K$ band. Despite the generally good agreement of the models with the individual bands, the inferred gravities and effective temperatures disagree depending on the spectral region being considered. Two regions of local minima are clearly visible for the effective temperature, one at $\approx 1500-1700 \mathrm{~K}$ and one at $\approx 2600-2900 \mathrm{~K}$. For the entire spectrum, the best-fitting model is visually a poor match to the data despite formally producing the lowest $\chi_{\nu}^{2}$ value. Similarly, the inferred radii span 1.7-5.1 $R_{\text {Jup }}$ depending on the spectral bandpass used in the fits. Only the $H$ and $K$ bands are consistent with expectations of $\approx 2 R_{\text {Jup }}$ from hot-start evolutionary models. The implied masses based on surface gravity and radii are also unphysical, ranging from $3.8 M_{\mathrm{Jup}}$ for the $H$ band to $3300 M_{\text {Jup }}$ for the entire spectral fit. These discrepancies make it difficult to interpret the results; systematic errors in the models mean that the data are not standard deviates about the model, and therefore the $\chi_{\nu}^{2}$ statistic does not correspond to a maximum likelihood. Instead, these fits are more sensitive to the overall shape of the pseudocontinuum rather than individual absorption features that may be more useful for constraining surface gravity and effective temperature.

For this reason, we explored another approach based on maximizing the cross-correlation between models and data. This method better incorporates information from atomic and molecular lines, which may be more sensitive to temperature and surface gravity, compared with the $\chi^{2}$ treatment. Crosscorrelation encodes line positions and line ratios but is mostly insensitive to uncertainties in the calibration of the continuum, as well as broadband spectral variations (e.g., Brogi et al. 2012). 
Table 4

K I Equivalent Widths of Young PMCs

\begin{tabular}{|c|c|c|c|c|c|c|}
\hline Object & SpT & $\begin{array}{c}\text { K I } 1.169 \mu \mathrm{m} \\
\text { EW }(\AA)\end{array}$ & $\begin{array}{c}\text { K I } 1.177 \mu \mathrm{m} \\
\mathrm{EW}(\AA)\end{array}$ & $\begin{array}{c}\text { K I } 1.243 \mu \mathrm{m} \\
\text { EW }(\AA)\end{array}$ & $\begin{array}{c}\text { K I } 1.253 \mu \mathrm{m} \\
\text { EW }(\AA)\end{array}$ & Reference \\
\hline GSC 6214-210 B & M9.5 \pm 1 & $\ldots$ & $\ldots$ & $3.0 \pm 0.5$ & $3.2 \pm 0.2$ & (1) \\
\hline ROXs 12 B & $\mathrm{L} 0 \pm 2$ & $1.10 \pm 0.09$ & $2.60 \pm 0.10$ & $0.88 \pm 0.04$ & $1.26 \pm 0.05$ & (2) \\
\hline ROXs 42B b & $\mathrm{L} 1 \pm 1$ & $2.8 \pm 0.5$ & $3.12 \pm 0.19$ & $1.9 \pm 0.3$ & $1.76 \pm 0.16$ & (1) \\
\hline $2 \mathrm{M} 0441+2301 \mathrm{Bb}$ & $\mathrm{L} 1 \pm 1$ & $\ldots$ & $\ldots$ & $5.4 \pm 0.6$ & $4.0 \pm 0.6$ & (3) \\
\hline 1RXS J1609-2105 B & $\mathrm{L} 2 \pm 1$ & $\cdots$ & $\cdots$ & $5.33 \pm 1.0$ & $3.8 \pm 0.4$ & $(4),(5),(6)$ \\
\hline
\end{tabular}

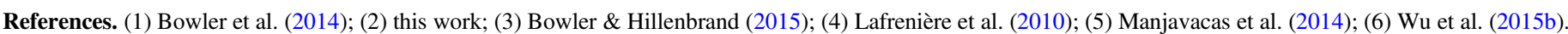
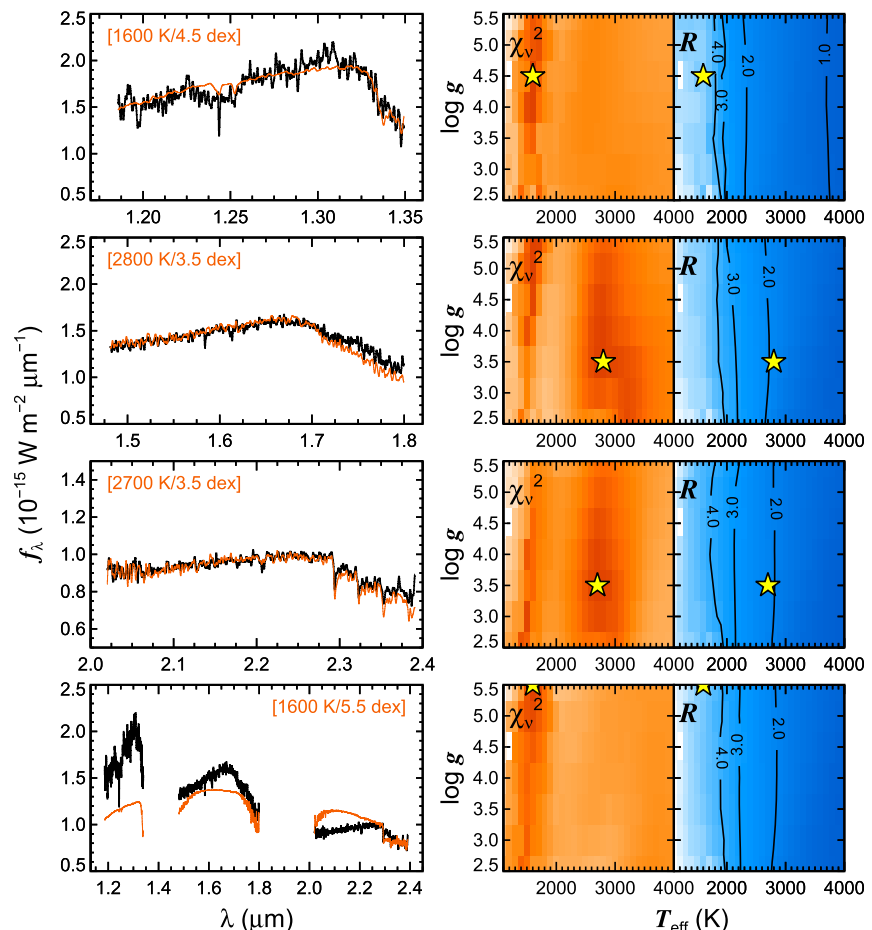

Figure 9. Best-fitting BT-Settl atmospheric models to our OSIRIS spectrum of ROXs 12 B. The top three panels show the results for the individual $J, H$, and $K$ bandpasses. The individual best-fitting models' effective temperature and surface gravity are given in red. The right panels show the reduced $\chi^{2}$ maps and associated radius in $R_{\text {Jup }}$ (using the scaling factor and distance to the system), with a star marking the global minimum.

The same set of models described above was convolved with a Gaussian kernel to a resolution of $R=10,000$. This is high enough to provide a high-enough resolution template for both the OSIRIS and the NIFS spectra but low enough to avoid interpolation errors. Prior to cross-correlation, a high-pass filter was applied to both the data and the models to remove broadband variations. The models were then Doppler-shifted to radial velocities between -900 and $+900 \mathrm{~km} \mathrm{~s}^{-1}$ (in steps of $30 \mathrm{~km} \mathrm{~s}^{-1}$ ), linearly interpolated to the spectral range of the data, and cross-correlated with our observed spectra. Each spectral channel in the data was weighted by the inverse of its relative error to prevent noisy sections of the spectra from dominating the analysis.

The cross-correlation functions (CCFs) obtained with the full set of model spectra are shown in Figure 10. Not only does the $\mathrm{S} / \mathrm{N}$ vary between different bands, but the peak of the crosscorrelation seems to occur at different positions in the spectral sequence, confirming the previous observation that different

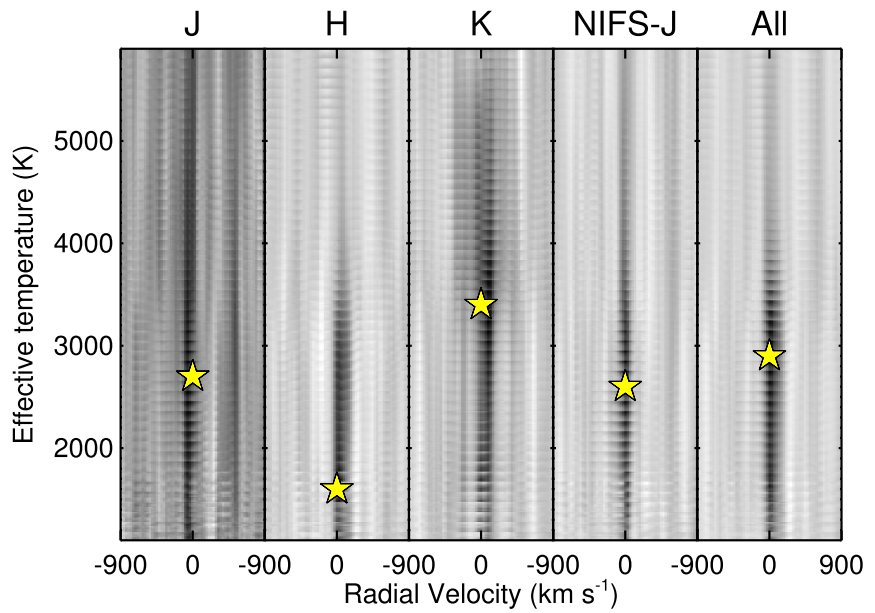

Figure 10. Cross-correlation plots for BT-Settl synthetic spectra relative to our OSIRIS and NIFS spectra of ROXs $12 \mathrm{~B}$. Each row shows the CCF for that particular model in velocity space, with higher cross-correlation values represented as darker pixel values. Each effective temperature is subdivided into rows with eight models spanning surface gravities between 2.0 and 5.5 dex, which produces the horizontal structure visible in each panel. The rightmost panel shows the cross-correlation results after combining the individual bandpasses. Yellow stars denote the maximum cross-correlation defined as having the highest $\mathrm{S} / \mathrm{N}$ value.

bands could lead to different best-fitting parameters for the atmosphere.

To formally compute confidence intervals (CIs) on the model parameters, we incorporated the values of the CCFs into a likelihood function $L$ following the approach of Zucker (2003). Since in this case we are combining four different bands, we adopt the following effective CCF:

$$
\bar{C}^{2}=1-\left\{\prod_{i}\left[1-C_{i}^{2}\right]\right\}^{1 / 4},
$$

where the product is performed over the CCF of the four available spectral bands. The effective $\mathrm{CCF}$ and the number of spectral channels $N$ are used to compute a log-likelihood function

$$
\log (L)=-\frac{N}{2} \log \left[1-\bar{C}^{2}\right],
$$

and the corresponding likelihood values $L$ are used to derive CIs.

The bottom left panel in Figure 11 shows the twodimensional map of $\log (L)$ (blue), and the corresponding $1 \sigma$, $2 \sigma$, and $3 \sigma$ CIs are labeled. The top left and bottom right panels 

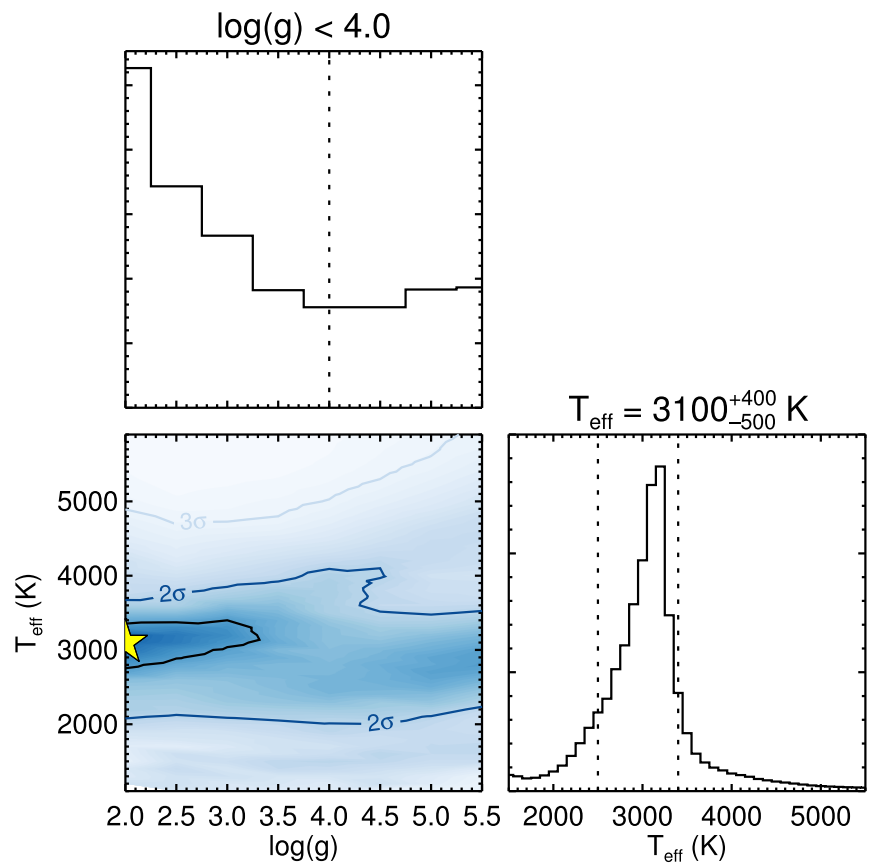

Figure 11. Cumulative cross-correlation results for all of our moderateresolution spectra of ROXs $12 \mathrm{~B}$. The bottom left panel shows the joint constraint on effective temperature and surface gravity, while the top left and bottom right panels display their projected one-dimensional distributions. The constraints are generally broad, with a $1 \sigma$ upper limit of 4.0 dex for the surface gravity and an effective temperature of $3100_{-500}^{+400} \mathrm{~K}$. However, as opposed to the $\chi^{2}$ approach (e.g., Figure 9), the bimodality is removed and robust confidence intervals can be obtained. The yellow star denotes the peak of the joint CCF.

show the projected probability distributions for $\log (g)$ and $T_{\text {eff }}$, respectively. The resulting effective temperature of ROXs $12 \mathrm{~B}$ is $T_{\text {eff }}=3100_{-500}^{+400} \mathrm{~K}$, and the bimodality observed with the previous model comparison is no longer present. However, only an upper limit of $\log (g)<4.0$ is obtained for the surface gravity. Although this result points to a low-gravity object, the likelihood also rises at high values of $\log (g)$.

The inferred effective temperature from our cross-correlation analysis is warmer than expected for a young L0 object, although the distribution is broad and encompasses the range of anticipated values. For example, using the effective temperature-spectral type relation for young objects from Filippazzo et al. (2015) yields $2260 \pm 60 \mathrm{~K}$, assuming no error on the spectral type. If we instead adopt an uncertainty of two subtypes, the median effective temperature of the resulting distribution is $2160 \mathrm{~K}$ with a $1 \sigma$ highest posterior density range ${ }^{14}$ of $1810-2770 \mathrm{~K}$.

We determine the luminosity of ROXs 12 B using our fluxcalibrated OSIRIS spectrum together with bolometric corrections using BT-Settl model synthetic spectra at shorter $(\lambda<1.2 \mu \mathrm{m})$ and longer $(\lambda>2.4 \mu \mathrm{m})$ wavelengths. The synthetic spectrum is scaled to the short- and long-wavelength endpoints of our spectrum and then integrated to derive a bolometric flux. Because the results from atmospheric model fits are either inconclusive (for the $\chi^{2}$ analysis) or have large uncertainties (from the $\mathrm{CCF}$ analysis), we utilize the $T_{\text {eff }}=2300 \mathrm{~K}$ model with $\log g=4.0$ dex based on relations

\footnotetext{
14 Also known as the minimum credible interval, this is the region containing the highest values of a posterior distribution and may comprise several intervals for multimodal distributions.
}

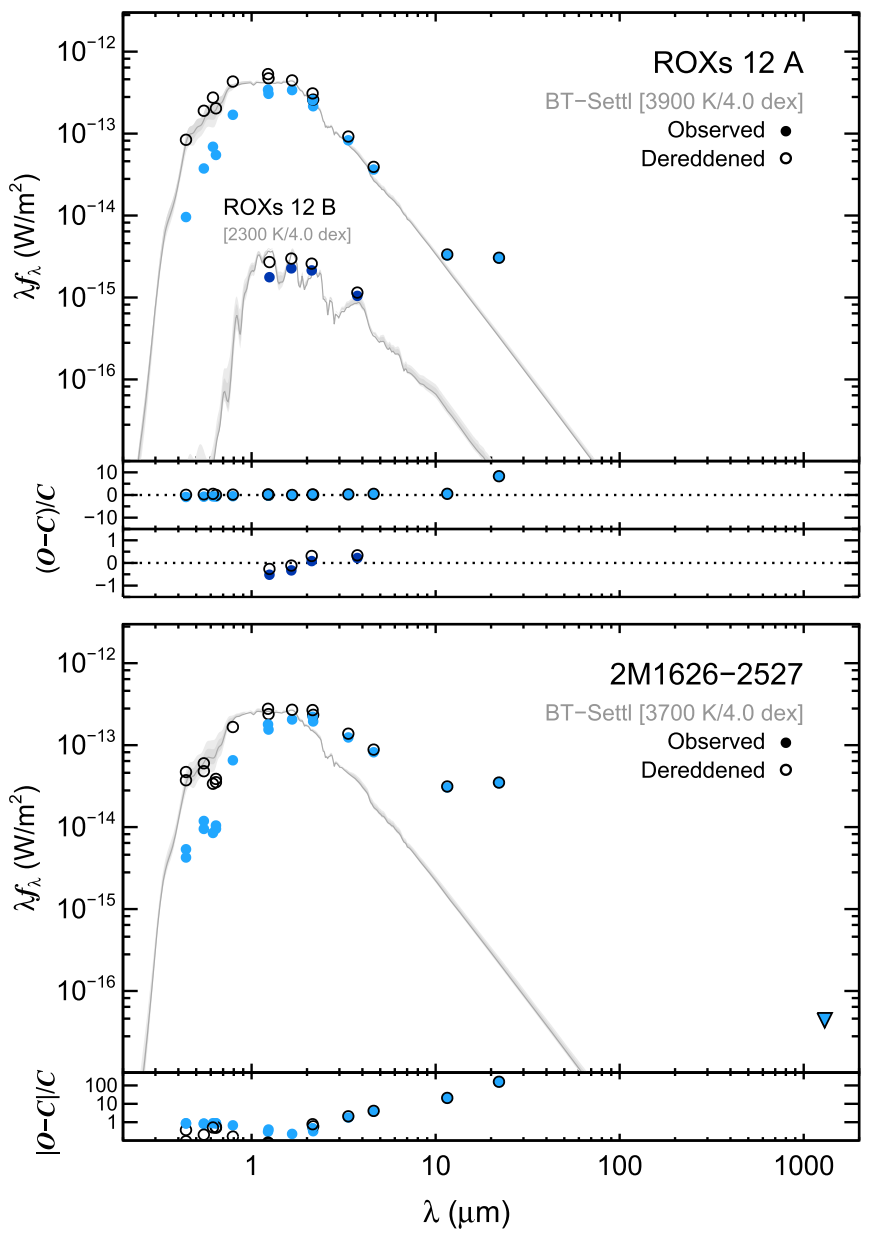

Figure 12. Spectral energy distributions for ROXs $12 \mathrm{~A}$, ROXs $12 \mathrm{~B}$, and 2M1626-2527. Raw photometry is shown as filled colored circles, while open circles have been dereddened by $A_{V}=1.8 \mathrm{mag}$. BT-Settl models are shown in dark gray with the nominal effective temperature and gravity of each object, as well as $\pm 100 \mathrm{~K}$ ranges shaded in gray. Models have been scaled to the dereddened $H$-band flux densities.

from Filippazzo et al. (2015) for young L0 objects-although the resulting luminosities are only weakly sensitive to the input model. The bolometric luminosity of ROXs $12 \mathrm{~B}$ is $\log L_{\text {bol }} / L_{\odot}=-2.87 \pm 0.06$ dex, which takes into account uncertainties in individual spectral measurements, the fluxcalibration scale factor, and the estimated distance to the system. In comparison, using a $3100 \mathrm{~K}$ model produces a similar bolometric luminosity of $-2.82 \pm 0.06$ dex.

Based on the bolometric luminosity of the companion and the system age ( $6_{-2}^{+3} \mathrm{Myr}$; Section 3.6), the inferred mass of ROXs $12 \mathrm{~B}$ is $17.5 \pm 1.5 M_{\text {Jup }}$ from Burrows et al. (1997) hotstart evolutionary models. This mass is consistent with the value of $16 \pm 4 M_{\text {Jup }}$ found by Kraus et al. (2014).

\subsection{M1626-2527: A Wide Tertiary Companion}

2M1626-2527 appears to constitute a wide tertiary to ROXs 12 AB separated by $37^{\prime \prime}$, or $5100 \mathrm{au}$, at a distance of about $140 \mathrm{pc}$. The proper motions of this pair from Hot Stuff for One Year (HSOY; Altmann et al. 2017) are consistent with one another:

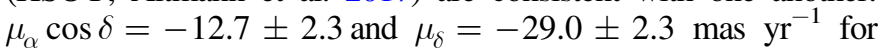
ROXs 12 and $\mu_{\alpha} \cos \delta=-11.9 \pm 2.3$ and $\mu_{\delta}=-30.5 \pm 2.3$ mas $\mathrm{yr}^{-1}$ for $2 \mathrm{M} 1626-2527$. Similarly, we measure consistent 

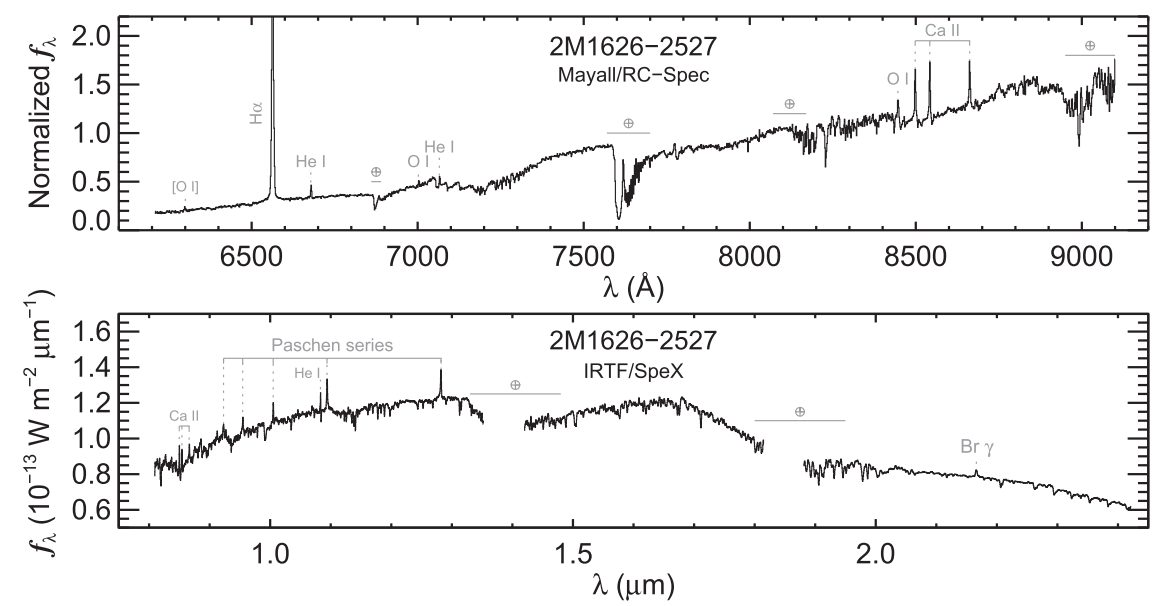

Figure 13. Optical and near-infrared spectra of 2M1626-2527 from Mayall/RC-Spec and IRTF/SpeX. Emission lines from He I, [O I], Ca II, He I, and $\mathrm{H}$ I-including strong $\mathrm{H} \alpha$ emission $(\mathrm{EW}=-46 \AA$ )—indicate active accretion onto this early-M dwarf. Note that strong veiling is evident at $\lesssim 7000 \AA$.

radial velocities for both components from our IGRINS spectra: $-6.09 \pm 0.16 \mathrm{~km} \mathrm{~s}^{-1}$ for ROXs 12 and $-6.03 \pm 0.16 \mathrm{~km} \mathrm{~s}^{-1}$ for 2M1626-2527. This is far less than the velocity dispersion of $\approx 1 \mathrm{~km} \mathrm{~s}^{-1}$ for $\rho$ Ophiuchus cluster members (Wilking et al. 2015), offering further evidence that these two stars are not simply members of the same cluster but are likely gravitationally bound. The identical radial velocities also suggest that both stars are probably single. Indeed, deep adaptive-optics imaging by Bryan et al. (2016) did not reveal any additional members of this system. The kinematic, photometric, and physical properties of this triple system comprising ROXs 12 A, ROXs 12 B, and 2M1626-2527 are listed in Table 2.

\subsection{Disk Properties and Accretion}

Figure 12 shows the spectral energy distributions of ROXs 12 A, ROXs 12 B, and 2M1626-2527 based on optical through infrared photometry in Table 2 . Flux-density measurements for all three targets are dereddened by $A_{V}=1.8 \mathrm{mag}$.

Solar metallicity BT-Settl model atmospheres from Allard et al. (2012) are overplotted in Figure 12. Spectral types are converted to effective temperatures using empirical calibrations for pre-main-sequence stars. ROXs $12 \mathrm{~A}$ has a spectral type of M0.0 \pm 0.5 (Bouvier \& Appenzeller 1992; Rizzuto et al. 2015), which corresponds to an effective temperature of $3900_{-90}^{+60} \mathrm{~K}$ on the Herczeg \& Hillenbrand (2014) scale and $3770_{-70}^{+100} \mathrm{~K}$ on the Pecaut \& Mamajek (2013) scale; we adopt an effective temperature of $3900 \pm 100 \mathrm{~K}$ for ROXs $12 \mathrm{~A}$ here. Without a large sample of radius measurements for young stars, it is difficult to assess the size of potential systematic errors in these spectral type-effective temperature scales. However, we note that even systematic errors as large as $300 \mathrm{~K}$ for ultracool dwarfs (e.g., Dupuy et al. 2010) do not substantially affect the broad results or conclusions regarding the luminosities, radii, or stellar inclinations throughout this paper.

The agreement of the model atmosphere with the dereddened photometry of ROXs 12 A is generally quite good, spanning the optical to mid-infrared wavelengths. But the SED clearly shows a large excess at $22 \mu \mathrm{m}$, presumably from disk emission originating from the host star ROXs 12 A or, less likely, a warm disk surrounding the companion ROXs 12 B that is only prominent at $22 \mu \mathrm{m}$. If this excess emission originates from ROXs $12 \mathrm{~A}$ and peaks at $\sim 22 \mu \mathrm{m}$, then the dust temperature is $\sim 230 \mathrm{~K}$ and is located at $\approx 0.7 \mathrm{au}$ following the heuristic relations from Wyatt (2008). If it originates from ROXs $12 \mathrm{~B}$, then it must be located much closer in, at $\sim 0.05$ au.

For ROXs $12 \mathrm{~B}$, the dereddened $J, H, K p$, and $L^{\prime}$ photometry from Kraus et al. (2014) is in good agreement with the $2300 \mathrm{~K}$ BT-Settl model, which was chosen based on spectral typeeffective temperature relations for young brown dwarfs (see Section 3.2). There is no evidence of excess emission from ROXs 12 B that would indicate that it hosts a circumsubstellar disk. This contrasts with many other young brown dwarfs and PMCs that harbor accretion subdisks, as evidenced by optical/ near-infrared emission lines and thermal infrared excess (e.g., Bowler et al. 2011; Bailey et al. 2013; Zhou et al. 2014).

No spectral type has been published for 2M1626-2527. Our own low-resolution optical spectrum shown in Figure 13 reveals a late-type spectrum with clear signs of active accretion, including strong $\mathrm{H} \alpha$ emission ( $\mathrm{EW}=-46 \AA$ ), as well as various transitions from $\mathrm{He} \mathrm{I},[\mathrm{O} \mathrm{I}$, and $\mathrm{Ca} \mathrm{II}$, all in emission (Table 5). Aside from $\mathrm{H} \alpha$ emission, the optical spectrum of $2 \mathrm{M} 1626-2527$ is relatively featureless shortward of $\approx 7000 \AA$ and appears to be heavily veiled. This likely originates from the boundary layer of an accretion disk (e.g., Basri \& Batalha 1990; Hartigan et al. 1991). The near-infrared spectrum of 2M1626-2527 shown in Figure 13 also points to a late (M-type) spectrum with strong emission lines from $\mathrm{Ca}$ II, $\mathrm{He} \mathrm{I}$, and $\mathrm{HI}$ (the Paschen series and $\mathrm{Br} \gamma$; see Table 5).

The relative depths of the $\mathrm{TiO}$ bandheads spanning 7050-7150 A are highly sensitive to temperature and can be used as an indicator of spectral type for 2M1626-2527 despite the heavy veiling at shorter wavelengths. Figure 14 shows a detailed view of this region compared to K4-M4 templates from Bochanski et al. (2007) for the M dwarfs and Mann et al. (2013) for the K dwarfs. The relatively pronounced $\mathrm{TiO}$ bands in 2M1626-2527 are stronger than the K4-M0 templates and weaker than the M2-M4 templates, with M1 being the best match. We therefore assign 2M1626-2527 a spectral type of M1 with an uncertainty of one subclass to reflect the imperfect agreement with this field template. The corresponding effective temperature is $3720_{-160}^{+180}$ and $3630 \pm 140 \mathrm{~K}$ using the Herczeg \& Hillenbrand (2014) and Pecaut \& Mamajek (2013) conversions, respectively. We adopt $T_{\text {eff }}=3700 \pm 150 \mathrm{~K}$ for 2M1626-2527. A model effective temperature of $3700 \mathrm{~K}$ and reddening of $A_{V}=1.8 \mathrm{mag}$ is a good fit to the photometry of 2M1626-2527 in the optical and near-infrared, but beyond 
Table 5

Emission-line Equivalent Widths

\begin{tabular}{|c|c|c|}
\hline $\begin{array}{l}\lambda_{0}{ }^{\mathrm{a}} \\
(\AA)\end{array}$ & $\begin{array}{c}\text { Line } \\
\text { ID }\end{array}$ & $\begin{array}{l}\text { EW } \\
(\AA)\end{array}$ \\
\hline \multicolumn{3}{|c|}{ ROXs 12 A: Keck/HIRES } \\
\hline 6562.8 & $\mathrm{H} \alpha$ & $-1.41 \pm 0.02$ \\
\hline 8498.0 & $\mathrm{Ca}$ II & $-0.40 \pm 0.01$ \\
\hline 8542.1 & $\mathrm{Ca}$ II & $-0.60 \pm 0.01$ \\
\hline 8662.1 & $\mathrm{Ca}$ II & $-0.42 \pm 0.02$ \\
\hline \multicolumn{3}{|c|}{ 2M1626-2527: Keck/HIRES } \\
\hline 4861.3 & $\mathrm{H} \beta$ & $-26.2 \pm 0.2$ \\
\hline 4921.9 & He I & $-0.53 \pm 0.03$ \\
\hline 5015.7 & He I & $-0.51 \pm 0.03$ \\
\hline 5875.6 & He I & $-2.54 \pm 0.03$ \\
\hline 6562.8 & $\mathrm{H} \alpha$ & $-53.17 \pm 0.13$ \\
\hline 6678.2 & He I & $-1.12 \pm 0.02$ \\
\hline $8446^{\mathrm{b}}$ & O I & $-0.47 \pm 0.04$ \\
\hline 8498.0 & $\mathrm{Ca}$ II & $-1.1 \pm 0.01$ \\
\hline 8542.1 & Ca II & $-1.29 \pm 0.02$ \\
\hline 8662.1 & Ca II & $-0.82 \pm 0.01$ \\
\hline \multicolumn{3}{|c|}{ 2M1626-2527: Mayall/RC-Spec } \\
\hline 6300.3 & [O I] & $-0.9 \pm 0.3$ \\
\hline 6562.8 & $\mathrm{H} \alpha$ & $-46.5 \pm 0.5$ \\
\hline 6678.2 & He I & $-1.33 \pm 0.12$ \\
\hline $7002^{\mathrm{b}}$ & O I & $-0.4 \pm 0.2$ \\
\hline 7065.2 & He I & $-0.9 \pm 0.3$ \\
\hline $8446^{\mathrm{b}}$ & O I & $-1.09 \pm 0.05$ \\
\hline 8498.0 & $\mathrm{Ca}$ II & $-2.00 \pm 0.05$ \\
\hline 8542.1 & $\mathrm{Ca}$ II & $-1.91 \pm 0.04$ \\
\hline 8662.1 & Ca II & $-1.46 \pm 0.04$ \\
\hline \multicolumn{3}{|c|}{ 2M1626-2527: IRTF/SpeX } \\
\hline $8446^{\mathrm{b}}$ & O I & $-0.7 \pm 0.3$ \\
\hline 8498.0 & $\mathrm{Ca}$ II & $-1.9 \pm 0.3$ \\
\hline 8542.1 & $\mathrm{Ca}$ II & $-1.6 \pm 0.2$ \\
\hline 8662.1 & $\mathrm{Ca}$ II & $-0.8 \pm 0.2$ \\
\hline 9546.2 & H I Pa8 $(\epsilon)$ & $-0.9 \pm 0.3$ \\
\hline 10049.8 & H I Pa7 $(\delta)$ & $-1.5 \pm 0.2$ \\
\hline 10830.3 & He I & $-1.1 \pm 0.2$ \\
\hline 10938.2 & H I Pa6 $(\gamma)$ & $-1.7 \pm 0.2$ \\
\hline 128218.1 & $\mathrm{~Pa} \beta$ & $-1.85 \pm 0.13$ \\
\hline 21661.2 & $\mathrm{Br} \gamma$ & $-1.5 \pm 0.2$ \\
\hline
\end{tabular}

Notes.

${ }^{\text {a }}$ Nominal air wavelength.

${ }^{\mathrm{b}}$ Lines are blended in our spectrum.

about $3 \mu \mathrm{m}$ there is clearly very strong excess emission from a protoplanetary disk (Figure 12).

A few selected regions of our HIRES spectra of ROXs 12 A and 2M1626-2527 are shown in Figure 15. ROXs 12 A shows relatively weak $\mathrm{H} \alpha$ emission $(\mathrm{EW}=-1.41 \pm 0.02 \AA$ ) considering its young age. This is similar to the value of $1.2 \AA$ found by Bouvier \& Appenzeller (1992) and $-2.00 \pm 0.02 \AA$ from Rizzuto et al. (2015). These values are consistent with the lower envelope of $\mathrm{H} \alpha$ emission for premain-sequence M0 stars spanning the youngest star-forming regions, like Taurus (Kraus et al. 2017), to somewhat older populations, like Upper Sco (Rizzuto et al. 2015). In our HIRES spectrum, 2M1626-2527 shows strong, broadened $\mathrm{H} \alpha$ emission $(\mathrm{EW}=-53.17 \pm 0.13 \AA$ ) comparable to our

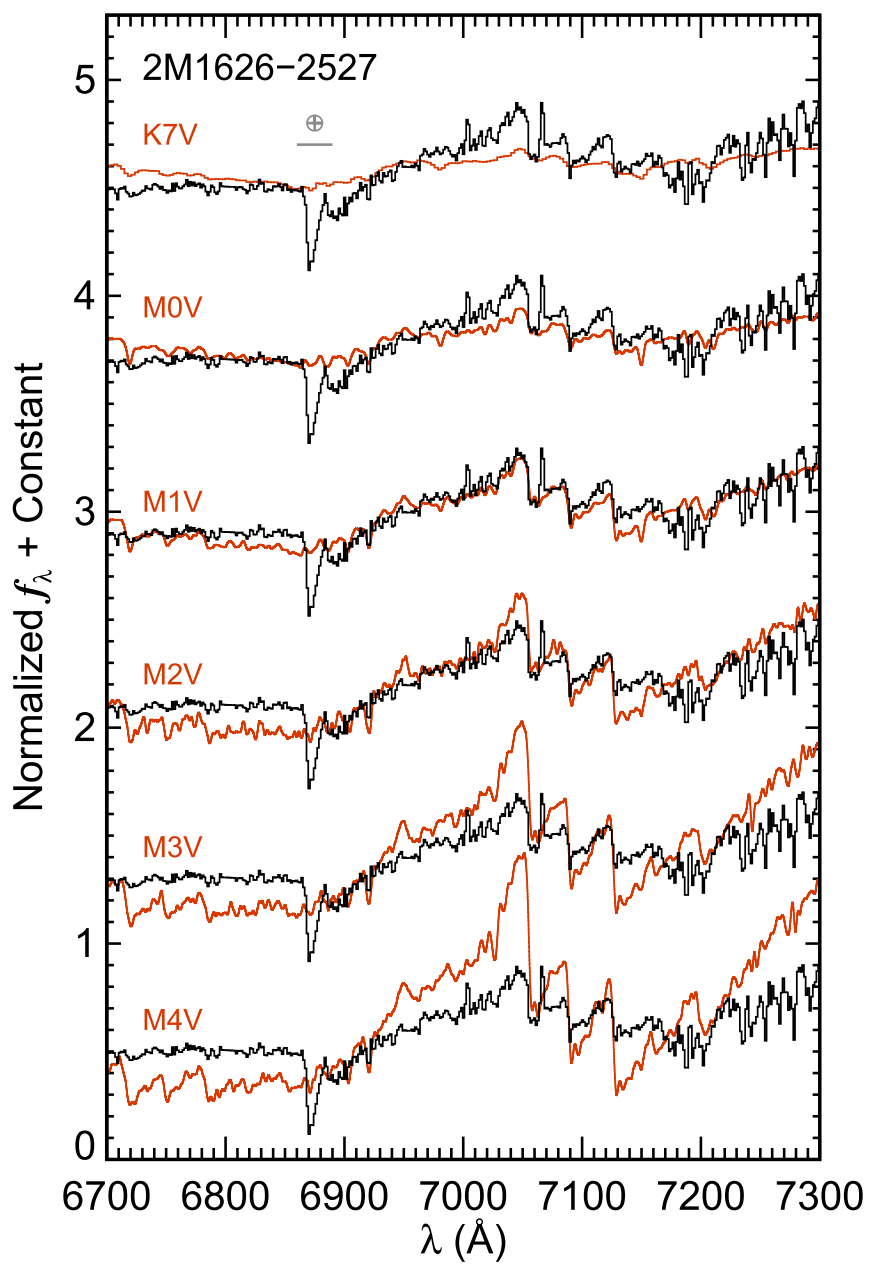

Figure 14. Comparison of our RC-Spec optical spectrum of 2M1626-2527 with K- and M-dwarf field templates from Mann et al. (2013) and Bochanski et al. (2007). The TiO bandheads between 7050 and $7150 \AA$ imply an early-M spectral type, with $\mathrm{M} 1$ being the best match. Note that the absorption feature at $\approx 6870 \AA$ is telluric $\left(\mathrm{O}_{2} B\right.$ band $)$.

measurement with RC-Spec $(\mathrm{EW}=-46.5 \pm 0.5 \AA)$. Both stars show Li I $6708 \AA$ absorption; we measure an equivalent width of $0.54 \pm 0.01 \AA$ for ROXs $12 \mathrm{~A}$-in good agreement with the value of $0.52 \pm 0.02 \AA$ from Rizzuto et al. (2015) and $\mathrm{EW}(\mathrm{Li})=0.32 \pm 0.02 \AA$ for $2 \mathrm{M} 1626-2527$. The Ca II infrared triplet is also seen in emission for both sources, with ROXs 12 A exhibiting broad absorption with superimposed central cores in emission. The Ca II line profiles from 2M1626-2527 show a broad, blueshifted component in emission, offset from the central narrow-line emission. This suggests that we are viewing a face-on accretion disk producing a broadened component along our line of sight together with a narrow zero-velocity peak where material hits the star. This geometry is consistent with the mostly pole-on stellar inclination we find for 2M1626-2527 in Section 4.2.

\subsection{K2 Light Curves}

The $K 2$ light curves for ROXs 12 A and 2M1626-2527 are shown in Figure 16. ROXs 12 A exhibits remarkably strong periodic changes with peak-to-peak semi-amplitude variations of $\approx 14 \%$. The most straightforward interpretation of these modulations is that they are caused by large, long-lived regions of 

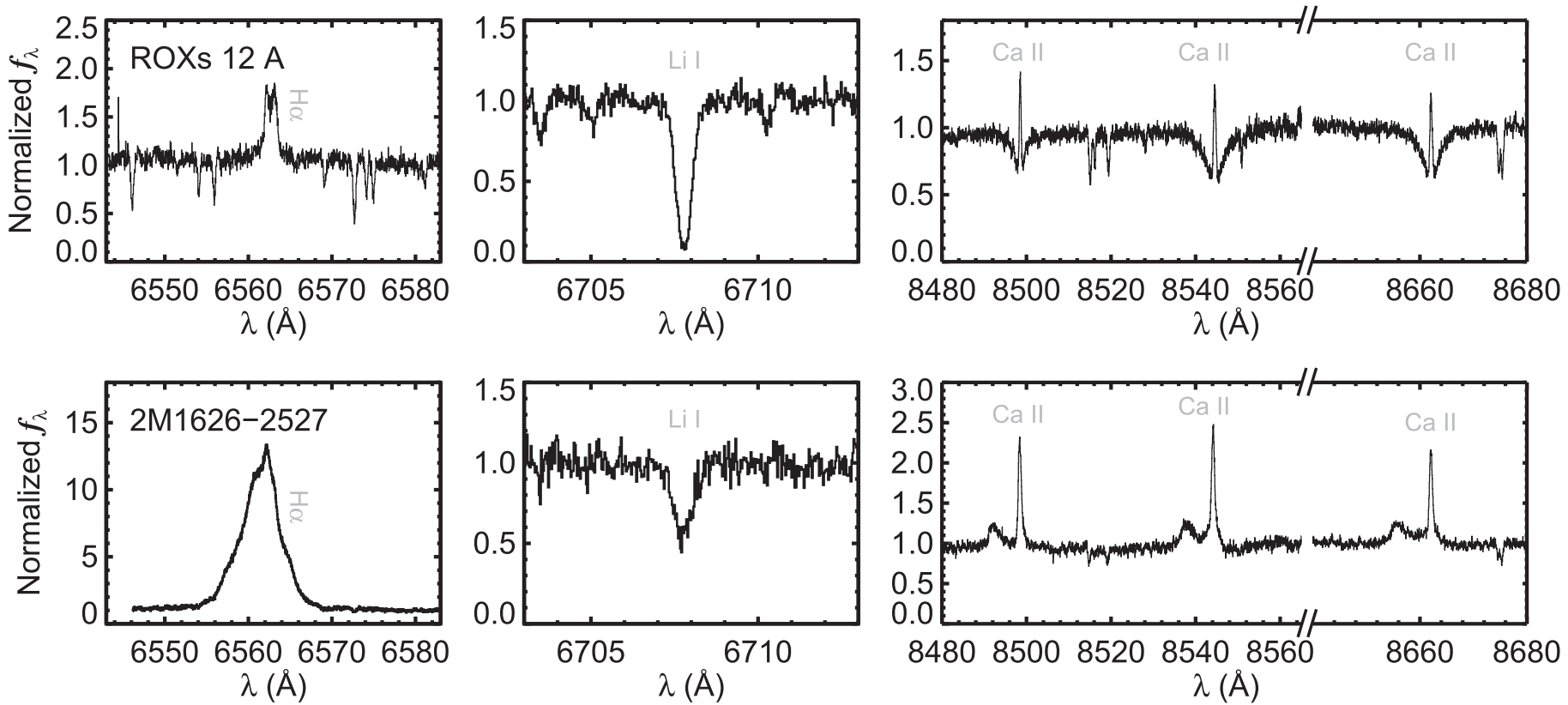

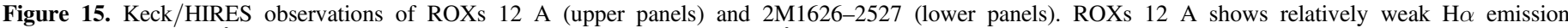

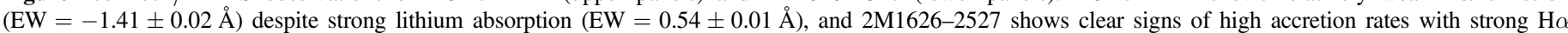
$(\mathrm{EW}=-53.17 \pm 0.13 \AA)$ and modest, possibly veiled lithium $(0.32 \pm 0.02 \AA)$. The calcium infrared triplet is in emission for both stars.
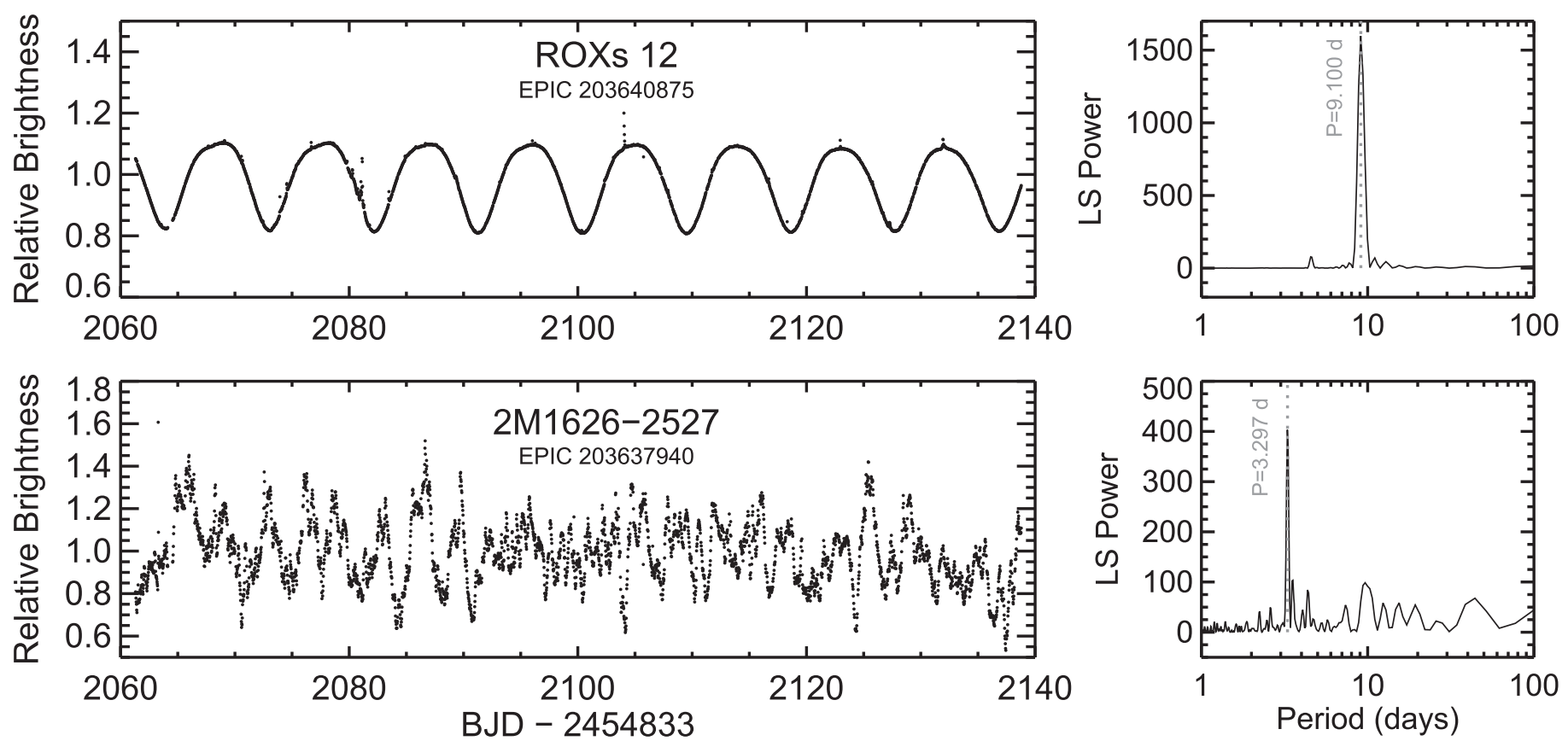

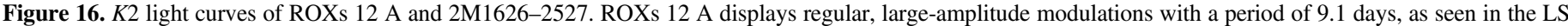

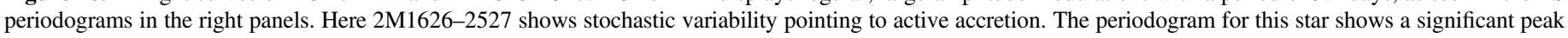
at 3.297 days, which we interpret as underlying rotational modulation. Phased light curves are plotted in Figure 17.

inhomogeneous spot coverage coming in and out of view as the star rotates. A Lomb-Scargle (LS) periodogram (Lomb 1976; Scargle 1982; Horne \& Baliunas 1986) reveals a strong peak period of 9.0998 days, which we adopt as the rotation period of ROXs 12 A. This is near the upper envelope of (but consistent with) rotation period measurements of low-mass pre-mainsequence stars during the first $\approx 1-10 \mathrm{Myr}$ of their evolution (e.g., Rebull et al. 2004; Irwin et al. 2011; Bouvier et al. 2014).
Several flaring events are clearly visible over the course of these observations. The $K 2$ light curve of ROXs 12 A phased to 9.100 days (Figure 17) exhibits only slight changes in the shape of the light curve from period to period, indicating minimal evolution of starspots on timescales less than one period.

The light curve of 2M1626-2527 shows both low-amplitude (few percent) and high-amplitude $(\approx 40 \%)$ variations on timescales ranging from hours to days (Figure 16). This 

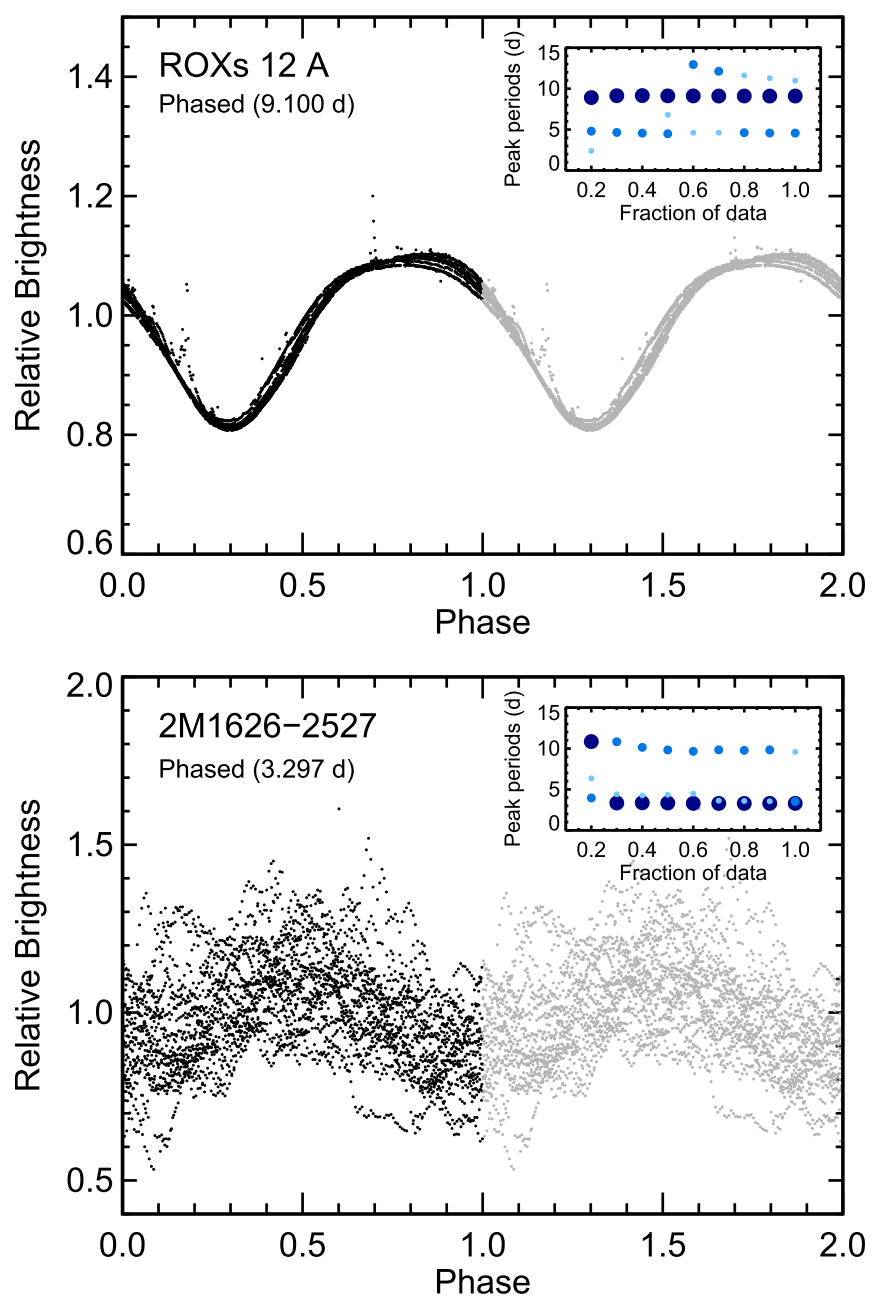

Figure 17. $K 2$ light curves of ROXs $12 \mathrm{~A}$ and $2 \mathrm{M} 1626-2527$ phased to the highest periodogram peak. ROXs 12 A shows slight changes from period to period, likely caused by slowly evolving starspots. Several flares are also evident. Here 2M1626-2527 is much more variable, but an underlying periodic signature is clearly visible in this phased curve. The inset panels for each star show the periods of the highest three periodogram peaks (large, medium, and small filled circles, respectively) as a function of the fraction of data being considered. For ROXs $12 \mathrm{~A}$, a consistent $\approx 9$ day period is the highest periodogram signal spanning the first $20 \%$ of the data out to the full light curve. For $2 \mathrm{M} 1626-2527$, the peak period for the first $20 \%$ of the data is at $\approx 11$ days, but it drops to $\approx 3.3$ days thereafter. This suggests that the underlying 3.3 day signal is probably not spurious, as it remains consistent over time.

stochastic behavior is characteristic of young accreting stars and is thought to be caused by time-dependent mass accretion events that produce transient hot spots on the stellar photosphere (e.g., Cody et al. 2014; Stauffer et al. 2016). Cody et al. (2017) found that this phenomenon is fairly common, occurring among $\approx 9 \%$ of $\rho$ Oph and Upper Sco members with strong infrared excesses. Although there are no obvious signs of periodicity in the light curve, the LS periodogram shows a significant peak power at 3.297 days. To explore whether this represents a real underlying modulation superimposed on stochastic variations, we applied the same periodogram analysis to smaller portions of the light curve, which should return the same approximate peak period near 3.3 days if the modulations are authentic and consistent over time. The inset plot in the lower panel of Figure 17 shows the peak period, second-highest peak, and third-highest peak starting with $20 \%$ of the light curve and progressively including more data in $10 \%$ bins. Beginning with $30 \%$ of the data, the peak periods range between 3.28 and 3.37 days. This suggests that the underlying modulations are real and are probably caused by rotationally modulated starspots. Interestingly, this means that 2M1626-2527 is rotating about three times faster than ROXs 12 A despite having an accreting disk, which has been shown, on average, to slow the rotation rates of young stars via stardisk interactions (e.g., Herbst et al. 2002; Cieza \& Baliber 2007). This is unusual but not entirely unexpected given the broad spread in periods for diskless and disk-bearing young stars.

We estimate the uncertainties for our light-curve periods following Kovács (1981) and Horne \& Baliunas (1986). For a single signal with Gaussian noise, they found that the error in the frequency measurement $\nu$ is $\delta \nu=3 \sigma /(4 \sqrt{N} T A)$, where $\sigma$ is the standard deviation of the noise about the signal, $N$ is the number of data points, $T$ is the period, and $A$ is the signal semiamplitude. From this, the period $(P)$ uncertainty is $\delta P=P^{2} \delta \nu$. We fit a sine curve to the phased data to find $A$ from the $K 2$ light curves. The rms noise was approximated by computing the standard deviation of the residuals between the time-series photometry and a Gaussian-smoothed version of the same data. From this analysis, we find formal uncertainties of 0.009 day for ROXS 12 and 0.002 day for 2M1626-2527. The high precision of these errors is primarily a result of the large number of periods sampled during the observing window (9-24 full cycles), the large number of data points in these light curves $(\approx 3400)$, and the relatively high semi-amplitudes for both systems $(10 \%-14 \%)$.

Stars exhibit differential rotation between their poles and equators, which can make it difficult to infer the equatorial rotation period from light curves alone if the location of their starspots is unknown. For example, the Sun has a longer rotation period of about 34 days at the poles compared to 25 days at its equator. Our period measurements for ROXs $12 \mathrm{~A}$ and 2M1626-2527 may therefore be overestimating the equatorial rotation periods of these objects, depending on in which latitudinal regions the starspots were located. Reinhold \& Gizon (2015) showed that the pole-equatorial shear $\left(\Delta \Omega=2 \pi\left(1 / P_{\min }-1 / P_{\max }\right)\right)$ of Kepler stars with effective temperatures comparable to those of ROXs $12 \mathrm{~A}$ and 2M1626-2527 ranges from about 0.01 to $0.10 \mathrm{rad} \mathrm{day}^{-1}$, with a typical value of about $0.03 \mathrm{rad} \mathrm{day}^{-1}$. This typical shear would imply a range of maximum and minimum periods of 8.7-9.5 days for ROXs 12 A and 3.25-3.35 days for 2M1626-2527. To take into account possible effects of differential rotation, we adopt larger rotation period uncertainties of $9.1 \pm 0.4$ days for ROXs 12 and $3.30 \pm 0.05$ days for 2M1626-2527 when using these values in the context of equatorial rotation period estimates.

\subsection{Stellar Luminosities, Masses, and Ages}

Bolometric luminosities for ROXs 12 A and 2M1626-2527 are derived using the 3900 and $3700 \mathrm{~K}$ BT-Settl models, respectively, shown in Figure 12. The models are first scaled to the $H$-band dereddened flux and then integrated from 0.2 to $200 \mu \mathrm{m}$ to find the bolometric flux. A distance of $137 \mathrm{pc}$ is assumed based on Very Large Baseline Array (VBLA) parallax measurements of Ophiuchus members in the Lynds 1688 dark cloud (Ortiz-León et al. 2017), and we adopt a \pm 10 pc uncertainty to encompass ambiguity in Ophiuchus versus Upper Sco membership. Uncertainties are derived by integrating models $100 \mathrm{~K}$ warmer and 100 

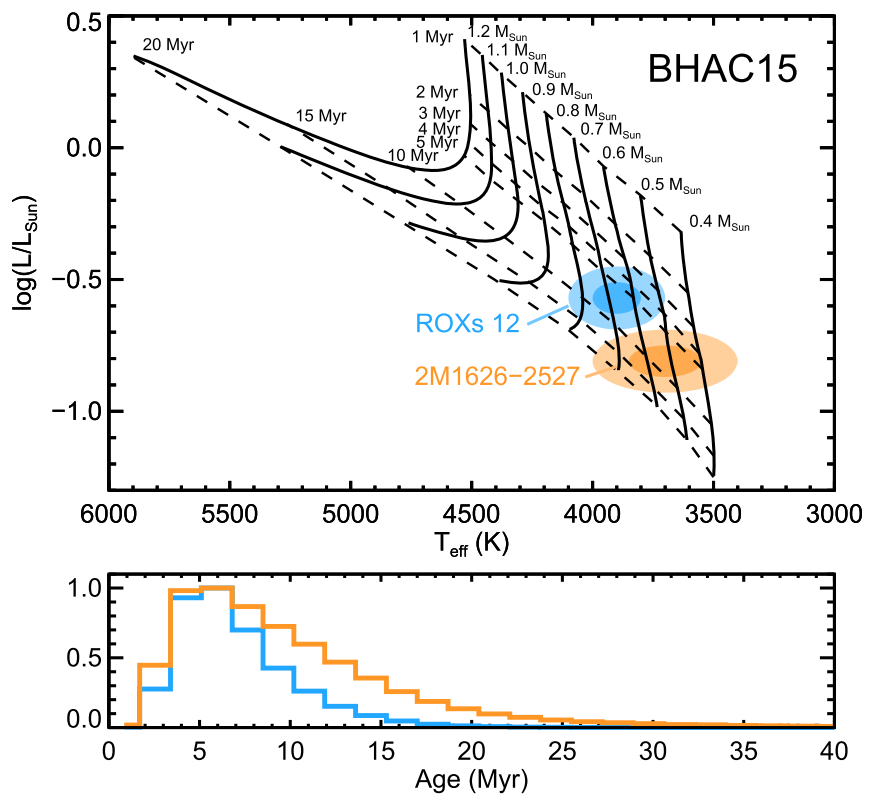

Figure 18. Comparison of ROXs 12 A (blue) and 2M1626-2527 (orange) to Baraffe et al. (2015) evolutionary models. Upper panel: H-R diagram showing the location of both stars from their effective temperatures and bolometric luminosities. Dark shaded ellipses show the $1 \sigma$ confidence regions; light shaded ellipses show the $2 \sigma$ regions. The corresponding masses from these models are $0.65_{-0.09}^{+0.05}$ and $0.5_{-0.1}^{+0.1} M_{\odot}$ for ROXs $12 \mathrm{~A}$ and $2 \mathrm{M} 1626-2527$, respectively. Lower panel: age distributions for both stars from their positions on the H-R diagram. The inferred age for ROXs $12 \mathrm{~A}$ is $6_{-2}^{+4} \mathrm{Myr}$, and for $2 \mathrm{M} 1626-2527$ it is $8_{-4}^{+7}$ Myr. Distributions have been normalized to their peak values for visual purposes.

$\mathrm{K}$ cooler than the nominal stellar effective temperatures and calculating the mean difference between these and the stars' bolometric fluxes. Surface gravities of $\log g=4.0$ dex are chosen based on expectations from evolutionary models (e.g., Baraffe et al. 2015), as substantial deviations from this are unphysical. This yields luminosities of $\log L_{\mathrm{bol}} / L_{\odot}=-0.57 \pm 0.06 \mathrm{dex}$ for ROXs $12 \mathrm{~A}$ and $-0.81 \pm 0.06$ dex for $2 \mathrm{M} 1626-2527$.

Stellar masses and ages are estimated using Baraffe et al. (2015) evolutionary models. For a given effective temperature and luminosity, we identify the corresponding mass and age by finely interpolating the grid of evolutionary models (Figure 18). This process is repeated in a Monte Carlo fashion to build a distribution of masses and ages assuming normally distributed errors in $T_{\text {eff }}$ and $\log \left(L_{\mathrm{bol}} / L_{\odot}\right)$. We infer a mass of $0.65_{-0.09}^{+0.05}$ $M_{\odot}$ and an age of $6_{-2}^{+4} \mathrm{Myr}$ for ROXs $12 \mathrm{~A}$. For 2M1626-2527, we find a mass of $0.50_{-0.10}^{+0.10} M_{\odot}$ and an age of $8_{-4}^{+7} \mathrm{Myr}$.

\section{Discussion}

\subsection{Ophiuchus or Upper Sco?}

The question of whether this system belongs to the younger Ophiuchus association or the older, more expansive Upper Sco region has implications for the inferred mass of the substellar companion ROXs $12 \mathrm{~B}$. ROXs 12 has historically been regarded as a member of the $\approx 0.5-2 \mathrm{Myr}$ Ophiuchus star-forming region since its identification by Bouvier \& Appenzeller (1992). This region is centered on the dark cloud Lynds 1688 , and its $>300$ members comprise a range of evolutionary stages spanning embedded protostars, accreting $\mathrm{T}$ Tauri stars, transition disks, and an older surface population that merges with the $\approx 5-10$ Myr Upper Sco subgroup (Wilking et al. 2008). ROXs
12 and 2M1626-2527 are located about 0.4 from the L1688 cloud core in (or behind) an extended region of modest extinction $\left(A_{V}=1-2\right.$ mag; Cambrésy 1999).

Unfortunately, because the internal velocity dispersions of cluster members are $\approx 1.0 \mathrm{~km} \mathrm{~s}^{-1}\left(\approx 1.5 \mathrm{mas} \mathrm{yr}^{-1}\right.$; Kraus \& Hillenbrand 2008; Wilking et al. 2015), the kinematics of Ophiuchus and Upper Sco are nearly indistinguishable using current proper-motion surveys. Mamajek (2008) found a mean proper motion of $\mu_{\alpha} \cos \delta=-10 \pm 1.5$ and $\mu_{\delta}=-27 \pm 1.5$ mas $\mathrm{yr}^{-1}$ (1 mas $\mathrm{yr}^{-1}$ systematic error) for Ophiuchus cloud members. Based on the $U V W$ space velocities of Upper Sco from Rizzuto et al. (2011), the expected proper motion of an Upper Sco member at the sky position of ROXs 12 is $\mu_{\alpha} \cos \delta=-11.9 \pm 1.5$ and $\mu_{\delta}=-24.4 \pm 1.5$ mas $\mathrm{yr}^{-1}$. (Note that because of the large angular extent of Upper Sco on the sky, the proper motions of its members vary slightly across this region, so we use the association's threedimensional space velocity at the sky position of ROXs 12 to calculate the expected proper motion.) The measured proper motions of ROXs 12 A and 2M1626-2527 (Table 2) agree with both of these regions to within about $2 \sigma$. Unsurprisingly, we find Sco-Oph membership probabilities of $99 \%$ for both stars following the Bayesian membership analysis described in Rizzuto et al. (2011, 2015).

Regardless of kinematics, the ages inferred for ROXs 12 A and 2M1626-2527 from their positions on the H-R diagram are most consistent with Upper Sco. Pecaut \& Mamajek (2016) adopted a median age of $10 \pm 3 \mathrm{Myr}$ for Upper Sco with a large intrinsic age spread of $\pm 7 \mathrm{Myr}$, which is consistent with recent findings by Fang et al. (2017). Pecaut et al. also found evidence of an age gradient in this subgroup, with ROXs 12 positioned near the transition point between $\approx 5$ and 9 Myr ages in the northwest and $\approx 11$ and $15 \mathrm{Myr}$ ages in the southeast. Our independently inferred ages for ROXs $12 \mathrm{~A}\left(6_{-2}^{+4} \mathrm{Myr}\right)$ and 2M1626-2527 ( $8_{-4}^{+7}$ Myr) from Section 3.6 (also see Figure 18) appear to agree with typical ages of Upper Sco members in the vicinity of the Ophiuchus cloud. In the near future, this membership analysis and age will be refined with parallactic distances and precise proper motions from Gaia.

\subsection{Stellar Radii and Line-of-sight Inclinations}

The radii of ROXs $12 \mathrm{~A}$ and 2M1626-2527 can be determined with the Stefan-Boltzmann law using their effective temperatures and bolometric luminosities. This yields values of $1.14 \pm 0.09 R_{\odot}$ for ROXs $12 \mathrm{~A}$ and $0.96 \pm 0.10 R_{\odot}$ for 2M1626-2527, where uncertainties are propagated analytically.

A lower limit on the radius of ROXs $12 \mathrm{~A}$ can also be inferred from the measured rotation period $\left(P_{\text {rot }}\right)$ and projected rotational velocity $\left(v_{p}=v \sin i_{*}\right)$. By equating $v$ and $2 \pi R_{*} / P_{\text {rot }}$, the stellar radius $R_{*}$ and associated uncertainty $\sigma_{R_{*}}$ are

$$
\begin{gathered}
R_{*}\left(i_{*}\right)=\frac{P_{\mathrm{rot}} v_{p}}{2 \pi \sin i_{*}} \\
\sigma_{R_{*}} \approx R_{*}\left(\left(\frac{\sigma_{P_{\mathrm{rot}}}}{P_{\mathrm{rot}}}\right)^{2}+\left(\frac{\sigma_{v_{p}}}{v_{p}}\right)^{2}+\left(\sigma_{i_{*}} \frac{\cos i_{*}}{\sin i_{*}}\right)^{2}\right)^{1 / 2} .
\end{gathered}
$$

In cases where the inclination is unknown, Equation (3) becomes a lower limit on $R_{*}$ if an edge-on orbit is assumed $\left(i_{*}=90^{\circ}\right)$. Adopting $P_{\text {rot }}=9.1 \pm 0.4$ days and $v_{p}=8.2 \pm 0.7 \mathrm{~km} \mathrm{~s}^{-1}$ 

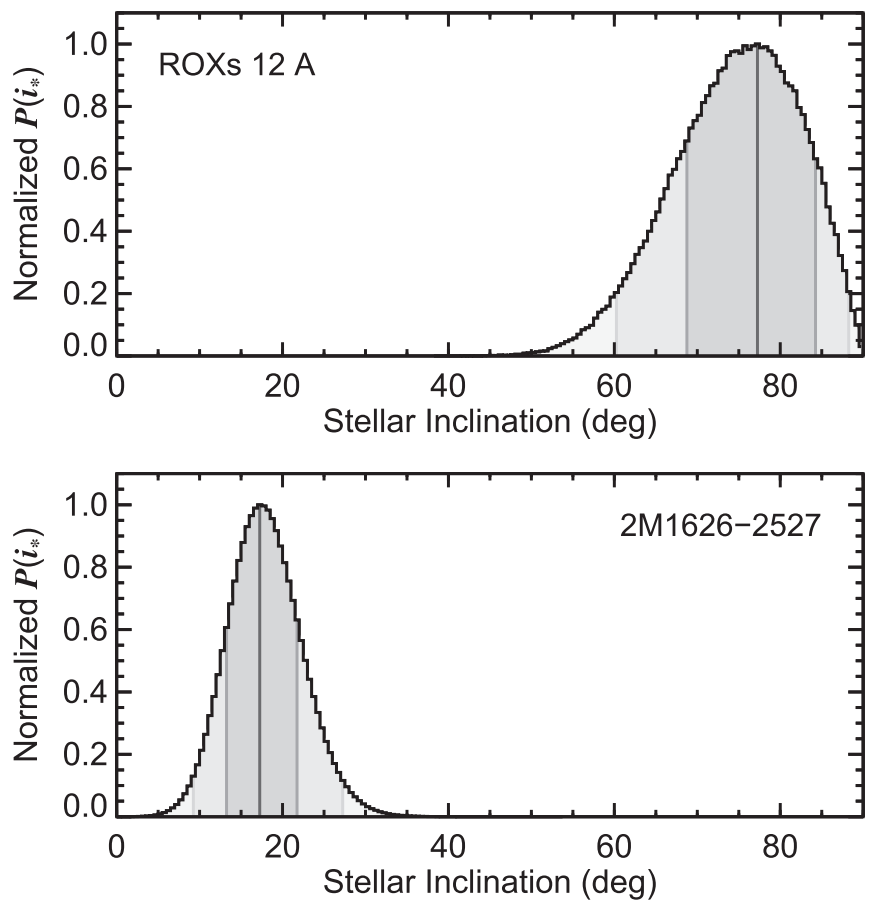

Figure 19. Constraints on the line-of-sight inclination of ROXs $12 \mathrm{~A}$ and 2M1626-2527 based on the stars' rotation periods, projected rotational velocities, effective temperatures, and bolometric luminosities (Equation (5)). ROXs $12 \mathrm{~A}$ is viewed mostly edge-on, with an inclination distribution that peaks at $77^{\circ}$ and a $95.4 \%$ highest posterior density interval of $60^{\circ}-88^{\circ}$. Here $2 \mathrm{M} 1626-2527$ is viewed mostly pole-on, with an inclination distribution that peaks at $17^{\circ}$ and a $95.4 \%$ highest posterior density interval of $9^{\circ}-27^{\circ}$.

for ROXs $12 \mathrm{~A}$ implies $R_{*}>1.47 \pm 0.14 R_{\odot}$. There is tension between this radius and the radius implied from the StefanBoltzmann law at the $2.0 \sigma$ level. The most likely origin of this discrepancy is that (1) the inferred effective temperature for ROXs $12 \mathrm{~A}$ is too high, (2) our $v \sin i_{*}$ measurement is too high, and/or (3) the rotation period samples high-latitude rather than equatorial starspots.

The line-of-sight inclination of ROXs 12 A can be determined more precisely using joint constraints from the "spectroscopic" (Stefan-Boltzmann) radius and the "rotational" $\left(R_{*}\left(i_{*}\right)\right)$ radius. Equating these two radii and solving for the stellar inclination gives

$$
i_{*}=\sin ^{-1}\left(\sqrt{\frac{\sigma_{\mathrm{SB}}}{\pi L_{\mathrm{bol}}}} v_{p} P_{\mathrm{rot}} T_{\mathrm{eff}}^{2}\right),
$$

where $\sigma_{\mathrm{SB}}$ is the Stefan-Boltzmann constant. Uncertainties are incorporated in a Monte Carlo fashion to build a line-of-sight inclination distribution, which peaks at $77^{\circ}$ and is truncated at $90^{\circ}$ (Figure 19). Monte Carlo realizations that result in $\sin i_{*}$ values $>1$ are excluded. The mode and $68.3 \%$ ( $1 \sigma$ equivalent) highest posterior density interval is $77_{-9}^{+7 \circ}$. The $95.4 \%(2 \sigma$ equivalent) range spans $60^{\circ}-88^{\circ}$. Note that these line-of-sight stellar inclinations are symmetric about $90^{\circ}$ and produce mirrored observational signatures at higher inclinations.

Similarly, using $P_{\text {rot }}=3.30 \pm 0.05$ days and $v_{p}=4.5 \pm$ $1.0 \mathrm{~km} \mathrm{~s}^{-1}$ for 2M1626-2527 implies $R_{*}>0.29 \pm 0.07 R_{\odot}$. This agrees with the spectroscopic radius of $0.96 \pm 0.10 R_{\odot}$. The joint constraint on the inclination distribution for $2 \mathrm{M} 1626-2527$ is shown in Figure 19. The mode and $68.3 \%$ highest posterior density interval is $17_{-4}^{+5 \circ}$, and the $95.4 \%$ range spans $9^{\circ}-27^{\circ}$.
The difference between these two inclination distributions, $\Delta i_{*}$, is the degree to which these two stars are misaligned. For ROXs $12 \mathrm{~A}$ and $2 \mathrm{M} 1626-2527$, we find $\Delta i_{*}=60_{-11}^{+7}$, indicating that these stars are strongly misaligned. This is not surprising for two young stars separated by over $5000 \mathrm{au}$, and it adds to mounting evidence that misalignments between the components of wide binaries is a common phenomenon (e.g., Hale 1994; Jensen \& Akeson 2014; Williams et al. 2014). This indicates that either the initial fragmenting cloud core that produced this system did not act as a uniformly corotating collapsing body, that this wide companion was captured, or that the rotational inclinations of these systems caused them to evolve with time.

\subsection{Obliquity of ROXs $12 A B$}

ROXs 12 B has a projected separation of 240 au, which corresponds to an orbital period of $4600 \mathrm{yr}$ and angular orbital motion of $0.08 \mathrm{yr}^{-1}$ assuming a circular face-on orbit. Because of the long baseline since the initial discovery epoch of ROXs 12 B by Ratzka et al. (2005) in 2001, enough time elapsed for Kraus et al. (2014) and Bryan et al. (2016) to detect small but significant orbital motion from this companion. Despite the small amount of orbital coverage, Bryan et al. (2016) were able to constrain the orbital elements of ROXs $12 \mathrm{~B}$ using the efficient rejection sampling algorithm for Keplerian orbits described in Blunt et al. (2017).

Of particular interest for this study is the posterior distribution of the orbital inclination, $i_{p}$, which peaks at about $135^{\circ}$ with a broad tail extending from about $90^{\circ}$ to $180^{\circ}$, as shown in Figure 20. The inclination of the stellar rotation axis of ROXs $12 \mathrm{~A}, i_{*}$, can be compared with $i_{p}$ to offer clues about the stellar obliquity - the relative orientation of the stellar spin axis and orbital angular momentum vector. The true (deprojected) angle between the stellar spin and planetary orbital axes, $\psi$, is related to the sky-projected spin-orbit angle, $\lambda$, as follows:

$$
\psi=\cos ^{-1}\left(\cos i_{*} \cos i_{p}+\sin i_{*} \sin i_{p} \cos \lambda\right) .
$$

Diagrams showing the geometric configuration can be found in, e.g., Ohta et al. (2005) and Fabrycky \& Winn (2009). The three angles $\lambda, i_{*}$, and $i_{p}$ must be known to determine $\psi$. The projected obliquity $(\lambda)$ is regularly measured for large transiting planets using the Rossiter-McLaughlin effect (e.g., Queloz et al. 2000; Winn et al. 2005; Johnson et al. 2009). In those cases, $i_{p} \approx 90^{\circ}$, so $\cos \psi \approx \sin i_{*} \cos \lambda$, and therefore the projected obliquity $(\lambda)$ is a measurement of the lower bound on the true obliquity $(\psi)$, assuming $i_{*}$ is unknown. Similarly, if $\lambda$ is unknown, as is the case for ROXs $12 \mathrm{AB}$, the lower limit on $\psi$ can be determined from the absolute difference between $i_{p}$ and $i_{*}: \psi \geqslant\left|i_{*}-i_{p}\right| \equiv \Delta i$ (see Appendix). Therefore, a system can have spin-orbit misalignment if $\Delta i$ is zero, but a nonzero value of $\Delta i$ means that the system must be misaligned by at least that amount (Glebocki \& Stawikowski 1997). In this sense, knowing both $i_{p}$ and $i_{*}$ offers comparable information about $\psi$, as do measurements of the Rossiter-McLaughlin effect.

The probability distribution functions for $i_{*}, i_{p}$, and $\Delta i$ for ROXs $12 \mathrm{~A}$ and $\mathrm{B}$ are shown in Figure 20. Note that since $i_{*}$ is symmetric about $90^{\circ}$, it mirrors Figure 19 and is therefore bimodal. The distribution of $\Delta i$ values peaks at $49^{\circ}$ with a $1 \sigma$ 

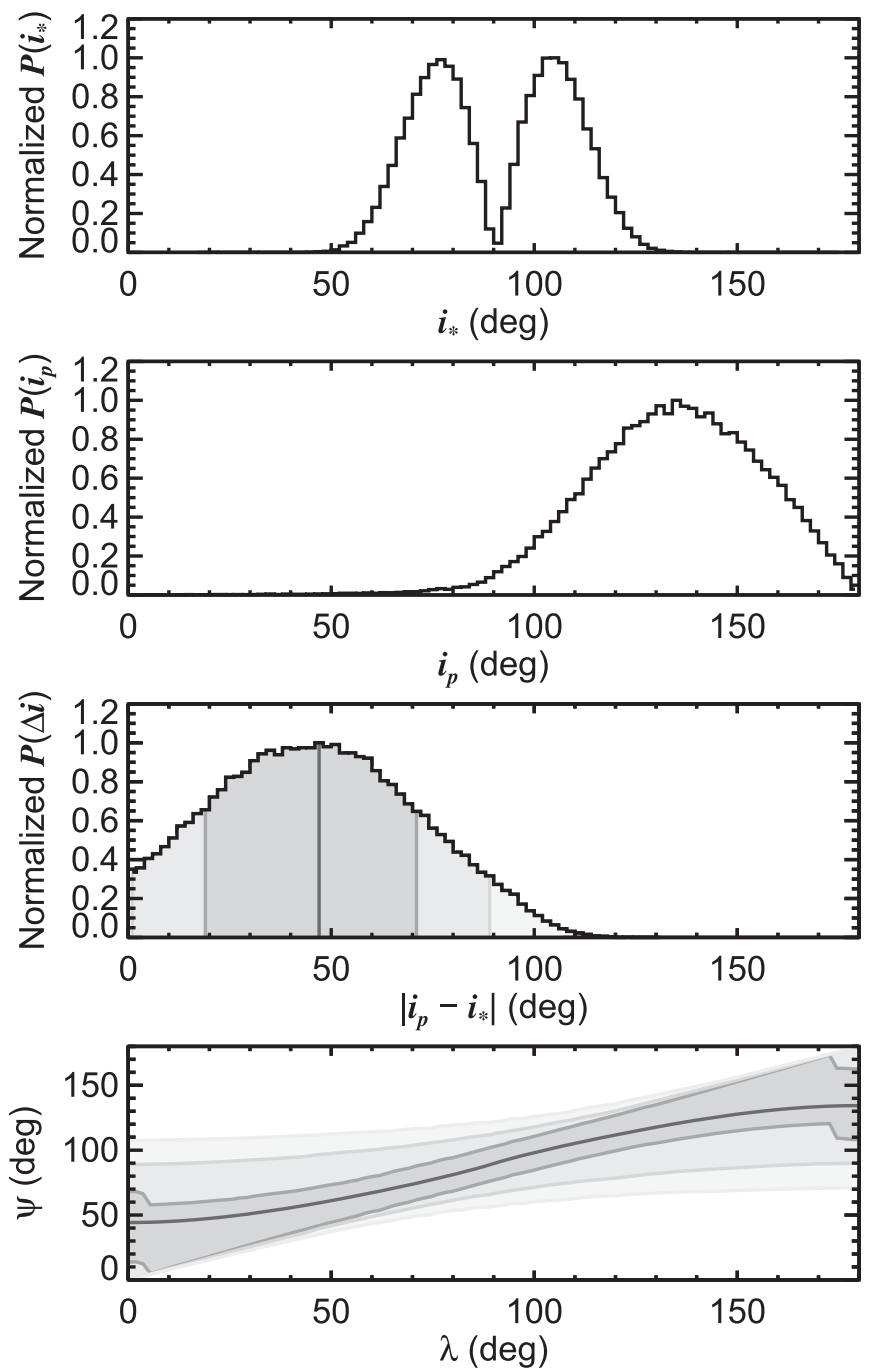

Figure 20. First panel: line-of-sight inclination $\left(i_{*}\right)$ of ROXs 12 A. Here we have mirrored the distribution from Figure 19 about $90^{\circ}$ owing to ambiguity in viewing geometry, which produces bimodal peaks. Second panel: orbital inclination $\left(i_{p}\right)$ of ROXs 12 B from Bryan et al. (2016) based on the measured orbital motion of the companion. Third panel: absolute difference between the stellar rotational axis and orbital axis of the companion $\left(\Delta i=\left|i_{p}-i_{*}\right|\right)$. This difference is a lower bound on the true deprojected obliquity angle $(\psi)$. The distribution peaks at $49^{\circ}$ with a $1 \sigma$ minimum confidence interval of $17^{\circ}-69^{\circ}$, indicating that ROXs $12 \mathrm{~A}$ and B likely exhibit spin-orbit misalignment. Fourth panel: contour plot demonstrating how the obliquity angle constraint varies as a function of the sky-projected spin-orbit angle $(\lambda)$ for the ROXs 12 $\mathrm{AB}$ system. The slice at $\lambda=0^{\circ}$ corresponds to the distribution presented in third panel. At greater values of (the unknown angle) $\lambda$, the distribution of $\psi$ steadily increases to higher values. Contours represent $1 \sigma, 2 \sigma$, and $3 \sigma$ minimum confidence intervals.

minimum CI of $17^{\circ}-69^{\circ}$. From this, we calculate that the probability that $\Delta i$ is greater than $10^{\circ}$ is $94 \%$. This distribution represents the minimum values of true obliquity angles in this system and indicates that ROXs $12 \mathrm{~A}$ and B are likely to have spin-orbit misalignment, although alignment $\left(\Delta i=0^{\circ}\right)$ cannot be ruled out from the observations.

\subsection{How Common is Pa $\beta$ Emission?}

The presence of disks around widely separated brown dwarfs and PMCs offers a convenient way to directly study the formation and late stages of growth of these objects. These subdisks produce a variety of observational signatures: ultraviolet excess emission from accretion of hot gas (Zhou et al. 2014), hydrogen-line emission like $\mathrm{H} \alpha$ and $\mathrm{Pa} \beta$ (e.g., Bowler et al. 2011; Wu et al. 2015a), and thermal excess emission in the mid-infrared (Bailey et al. 2013). Submillimeter emission from warm dust has only been detected for the ambiguous companion to FW Tau (Caceres et al. 2015; Kraus et al. 2015) despite ongoing searches targeting several other disk-bearing substellar companions (e.g., Bowler et al. 2015; MacGregor et al. 2017; Wu et al. 2017).

Although we find no convincing evidence that ROXs $12 \mathrm{~B}$ harbors a subdisk, the lack of $\mathrm{Pa} \beta$ emission in this individual object can nevertheless be used to more broadly assess the global frequency of low-mass companions with accretion rates large enough to result in $\mathrm{Pa} \beta$ line emission. The sample of young companions near and below the deuterium-burning limit that also have moderate-resolution $J$-band spectroscopy has increased over the past few years to the point where we can begin to quantify the statistical properties of these objects as a population. Note that the strength of the $\mathrm{Pa} \beta$ emission line is expected to vary strongly with effective temperature, which alters the pseudocontinnuum level, as well as mass accretion rate. In addition, both the $\mathrm{S} / \mathrm{N}$ and resolving power of a spectrum can influence the detection of an emission line. For this analysis, we ignore these effects and simply count reported $\mathrm{Pa} \beta$ detections and nondetections as discrete Bernoulli trials.

If we isolate a sample of young companions with ages $\lesssim 15 \mathrm{Myr}$, when such subdisks might still be expected to be actively accreting, and companion masses $\lesssim 20 M_{\text {Jup }}$, there are 11 low-mass brown dwarfs and planetary-mass objects with published moderate-resolution spectroscopy. Five of these show $\mathrm{Pa} \beta$ in emission: GSC 6214-210 B (Bowler et al. 2011; Lachapelle et al. 2015), CT Cha B (Schmidt et al. 2008; Bonnefoy et al. 2014), GQ Lup B (Seifahrt et al. 2007), DH Tau B (Bonnefoy et al. 2014; Wolff et al. 2017), and FW Tau b (Bowler et al. 2014). Six companions do not appear to have $\mathrm{Pa} \beta$ in emission: 1 RXS J1609-2105 B (Lafrenière et al. 2010), ROXs 42 Bb (Bowler et al. 2014), 2M0441+2301 Bb (Bowler \& Hillenbrand 2015), SR 12 C (Bowler et al. 2014), USco CTIO 108 B (Bonnefoy et al. 2014), and ROXs 12 B (this work). Binomial statistics implies a frequency of $46 \% \pm 14 \%$ for five detections out of 11 trials.

There are several caveats about these accreting companions that are worth noting. $\mathrm{Pa} \beta$ emission was not observed in the moderate-resolution spectroscopy of GQ Lup B presented by McElwain et al. (2007) and Lavigne et al. (2009), which suggests that the emission observed by Seifahrt et al. (2007) was variable. Similarly, Wolff et al. (2017) also found variable emission for DH Tau B. This suggests that other companions without $\mathrm{Pa} \beta$ emission could be observed during a quiescent state of low accretion. Published mass estimates for GQ Lup B range from $\sim 10$ to $40 M_{\mathrm{Jup}}$, so this companion is consistent with (but may fall above) the cutoff of $20 M_{\text {Jup }}$ we use in this analysis. Finally, the mass of FW Tau b is uncertain, as its spectrum shows substantial veiling and indications of an edgeon disk (Bowler et al. 2014).

Accretion subdisks are apparently very common among companions near the deuterium-burning limit. The incidence of both accreting and nonaccreting circumsubstellar disks in general is likely much higher-and perhaps universal-for these objects. 


\subsection{Implications of Misalignment for the Formation of ROXs $12 \mathrm{~B}$}

The three-dimensional orbital and rotational architecture of planetary systems provides fundamental insight into their formation and subsequent dynamical evolution. Massive giant planets ( $\gtrsim 1 M_{\text {Jup }}$ ) formed in circumstellar disks should inherit the orientation of the disks' angular momentum vectors, which are expected to initially be aligned with those of the host stars. Similarly, the orbital planes of planets should also align with the equatorial planes of host stars in the absence of internal (other planets) or external (wide binary companions or passing stars) perturbers. There is also now substantial evidence that the tail end of the star formation process can produce companions in the planetary-mass regime near the opacity limit for fragmentation (e.g., Todorov et al. 2010; Bowler \& Hillenbrand 2015). Just as binary companions that formed from the turbulent fragmentation of molecular cloud cores tend to have misaligned orientations (e.g., Brinch et al. 2016; Lee et al. 2016; Offner et al. 2016), the same random spin axes could also be imprinted on PMCs that form in this fashion. Together these two pathways-formation in a disk versus turbulent cloud core-have direct observational implications for wide substellar companions: in the absence of outside influences, massive isolated objects formed in a disk can be expected to exhibit spin-orbit alignment, whereas misalignment implies formation from turbulent fragmentation. Our result that ROXs $12 \mathrm{~B}$ is likely misaligned with the spin axis of its host star suggests that it was formed from the cloud fragmentation process, as opposed to disk instability, but this interpretation is somewhat complicated by the wide stellar tertiary, as discussed below.

It is also possible that ROXs $12 \mathrm{~B}$ could have formed from disk instability if it later underwent dynamical interactions with another body, for instance, through gravitational scattering or Kozai-Lidov librations with the wide stellar companion 2M1626-2527 (Kozai 1962; Lidov 1962; Naoz 2016). The timescale for Kozai-Lidov secular oscillations for a planet with an outer tertiary component is governed by the period of the planet, the masses of the host star and tertiary, the semimajor axes of the planet and tertiary, and the eccentricity of the hosttertiary orbit (e.g., Holman et al. 1997). For the ROXs 12 triple system, this characteristic timescale reduces to $\approx 6 \times 10^{7}$ $\left(1-e_{\text {ter }}^{2}\right)^{3 / 2} \mathrm{yr}$, where $e_{\text {ter }}$ is the eccentricity of the ROXs $12 \mathrm{~A}$ and 2M1626-2527 pair. As long as this eccentricity is below about 0.9 , oscillation timescales are likely too long to have substantially influenced the orbital inclination of ROXs $12 \mathrm{~B}$, given the young age of the system.

Similarly, prior to the planet formation process, wide stellar companions can gravitationally torque protoplanetary disks and result in spin-orbit misalignments with planets when they eventually form (e.g., Batygin 2012; Lai 2014). It is therefore conceivable that at a very early stage, a massive disk around ROXs 12 A could have been torqued by 2M1626-2527 to produce the misalignment we now observe in this system. For a protoplanetary disk around ROXs $12 \mathrm{~A}$, the disk precession period would be roughly 80 Myr following Batygin (2012) or about 20 Myr for maximal misalignment, assuming that the outer disk radius ends at the location of ROXs $12 \mathrm{~B}$. A more extended disk or a nonzero eccentricity for 2M1626-2527 can reduce this timescale even further, well within the age of $\approx 6 \mathrm{Myr}$ of this system, implying that the spin-orbit misalignment we observe for ROXs 12 B could plausibly be a result of an initial torque on a disk around ROXs 12 A caused by 2M1626-2527.

In addition to ROXs $12 \mathrm{~B}$, only a few other systems with widely separated substellar companions have information available about their stellar obliquity angles. There is some evidence that the orbital axis of the brown dwarf companion GQ Lup B may be misaligned with both the stellar rotation axis and the stellar disk inclination (Ginski et al. 2014; Schwarz et al. 2016; MacGregor et al. 2017; Wu et al. 2017), although it is unclear how significant this potential spin-orbit misalignment is due to discrepancies in the rotation period and radius of the host star. HR 8799 is an excellent example of a system with increasingly robust constraints on the spin axis of the host star, orbital inclination of its four imaged planets, and inclination measurements for its multibelt debris disk. Interestingly, each of these components appears to be mutually consistent within uncertainties (e.g., Reidemeister et al. 2009; Matthews et al. 2013; Booth et al. 2016; Konopacky et al. 2016), suggesting aligned angular momentum vectors and planet formation in a disk. As the orbits of directly imaged planets become better constrained in the future, stellar obliquity measurements like the one we have carried out here for ROXs $12 \mathrm{AB}$ will help identify the dominant formation pathway(s) for these objects as a population analogous to Rossiter-McLaughlin measurements for transiting planets.

\section{Summary}

We have carried out a comprehensive analysis of the ROXs 12 triple system comprising two young low-mass stars, ROXs $12 \mathrm{~A}$ and $2 \mathrm{M} 1626-2527$, and the $\approx 18 M_{\text {Jup }}$ companion ROXs 12 B. Our main results are summarized below.

1. Our moderate-resolution near-infrared spectra of ROXs 12 B show unambiguous signs of low surface gravity associated with youth. We find a spectral type of $\mathrm{LO} \pm 2$, a mass of $17.5 \pm 1.5 M_{\text {Jup }}$ based on hot-start evolutionary models, and an effective temperature of $3100_{-500}^{+400} \mathrm{~K}$ from cross-correlation with synthetic spectra of ultracool objects.

2. The distant stellar companion 2M1626-2527 shares consistent kinematics with the binary ROXs $12 \mathrm{AB}$, making this a wide $(\approx 5100 \mathrm{au})$ tertiary component. ROXs 12 A has a spectral type of $\mathrm{M} 0 \pm 0.5$, an effective temperature of $3900 \pm 100 \mathrm{~K}$, a mass of $0.65_{-0.09}^{+0.05} M_{\odot}$, and an inferred age of $6_{-2}^{+4} \mathrm{Myr} ; 2 \mathrm{M} 1626-2527$ has a spectral type of $\mathrm{M} 1 \pm 1$, an effective temperature of $3700 \pm 150 \mathrm{~K}$, a mass of $0.5 \pm 0.1 M_{\odot}$, and an inferred age of $8_{-4}^{+7}$ Myr. Both stars show lithium absorption. In addition, 2M1626-2527 hosts an actively accreting protoplanetary disk, whereas ROXs 12 A hosts a passive disk with no signs of accretion. Although both members of the pair are located near the young Ophiuchus starforming region, their older ages instead suggest that they are members of Upper Sco.

3. K2 light curves reveal a period of $9.1 \pm 0.4$ days for ROXs $12 \mathrm{~A}$ and $3.3 \pm 0.05$ days for 2M1626-2527. We combine our $v \sin i_{*}$ measurements of both stars with radius constraints to determine their line-of-sight inclinations. The rotation axes of these stars are misaligned by $60_{-11}^{+7}$, consistent with the pattern of random orientations found for other wide binaries. 
4. The orbit of ROXs $12 \mathrm{~B}$ is likely misaligned with the spin axis of its host star by at least $49_{-32}^{+20 \circ}$, suggesting formation via cloud fragmentation or possibly disk instability if the protoplanetary disk surrounding ROXs 12 A was gravitationally torqued by 2M1626-2527. ROXs $12 \mathrm{~B}$ is the lowest-mass imaged companion with evidence of spin-orbit misalignment. Continued orbit monitoring will better constrain the orbital inclination of the companion and lead to a more precise measurement of the host star's obliquity angle.

5. ROXs $12 \mathrm{~B}$ does not have $\mathrm{Pa} \beta$ emission or thermal ( $L$-band) excess, and we find no compelling evidence that it harbors a subdisk. However, as a population, the frequency of accreting subdisks is relatively high: $46 \% \pm 14 \%$ of companions with masses $\lesssim 20 M_{\text {Jup }}$ and ages $\lesssim 15$ Myr show $\mathrm{Pa} \beta$ emission. Since this emission is likely to be variable and some companions will not be accreting at all, this represents a lower limit on the occurrence rate of circumplanetary disks in general.

We thank the anonymous referee for constructive comments; we thank Trent Dupuy and Konstantin Batygin for helpful discussions about spin-orbit misalignments; Eric Nielsen and Sarah Blunt for the published posterior orbital inclination distribution of ROXs $12 \mathrm{~B}$; and the Keck and Gemini Observatory staff for their exceptional support. Support for this work was provided by the National Aeronautics and Space Administration (NASA) through Hubble Fellowship grants HST-HF2-51369.001-A and HST-HF2-51336.001-A awarded by the Space Telescope Science Institute, which is operated by the Association of Universities for Research in Astronomy, Inc. (AURA), for NASA, under contract NAS5-26555. H.A.K. acknowledges support from the Sloan Foundation. M.C.L. acknowledges support from National Science Foundation (NSF) grant NSF-AST-1518339. A.V. is supported by the NSF Graduate Research Fellowship, grant No. DGE 1144152. L.A.C. acknowledges grant support from CONICYT-FONDECYT number 1171246. This paper includes data taken at the McDonald Observatory of the University of Texas at Austin. It is also based on observations with the Mayall/RC-Spec spectrograph carried out through NOAO Prop. ID 2014A0019. Based on observations obtained at the Gemini Observatory (NOAO Prop. ID: 2016A-0112; Gemini Program ID: GN2016A-Q-37; PI: B Bowler; processed using the Gemini NIFS IRAF package), which is operated by AURA under a cooperative agreement with the NSF on behalf of the Gemini partnership: the NSF (United States); the National Research Council (Canada); CONICYT (Chile); Ministerio de Ciencia, Tecnología e Innovación Productiva (Argentina); and Ministério da Ciência, Tecnologia e Inovação (Brazil). IRAF is distributed by the National Optical Astronomy Observatory, which is operated by AURA under a cooperative agreement with the NSF. We utilized data products from the Two Micron All Sky Survey, which is a joint project of the University of Massachusetts and the Infrared Processing and Analysis Center/California Institute of Technology, funded by NASA and the NSF. NASA's Astrophysics Data System Bibliographic Services, together with the VizieR catalog access tool and SIMBAD database operated at CDS, Strasbourg, France, were invaluable resources for this work. This paper includes data collected by the Kepler mission. Funding for the Kepler mission is provided by the NASA Science Mission directorate.
This work used the Immersion Grating Infrared Spectrometer (IGRINS) that was developed under a collaboration between the University of Texas at Austin and the Korea Astronomy and Space Science Institute (KASI) with the financial support of the US National Science Foundation under grant AST1229522 to the University of Texas at Austin and of the Korean GMT Project of KASI. This publication makes use of data products from the Wide-field Infrared Survey Explorer, which is a joint project of the University of California, Los Angeles, and the Jet Propulsion Laboratory/California Institute of Technology, funded by NASA. Finally, mahalo nui loa to the kama'āina of Hawai'i for their support of Keck and the Maunakea observatories. We are grateful to conduct observations from this mountain.

Facilities: Keck:II (NIRC2), Keck:I (OSIRIS, HIRES), Smith (IGRINS), Gemini:Gillett (NIFS), Mayall (RC-Spec), Kepler, IRTF (SpeX).

\section{Appendix}

The two relevant angles that have been measured for this system are the line-of-sight inclination of ROXs 12 A's rotation axis, $i_{*}$, and the orbital inclination of ROXs $12 \mathrm{~B}, i_{p}$. These angles are related to the sky-projected obliquity $\lambda$ and the true (deprojected) spin-orbit obliquity $\psi$ via Equation (6). Here we show that the absolute difference between $i_{p}$ and $i_{*}$ is a lower limit on the measurement of $\psi$.

For values of $\lambda$ on the interval $\left[0^{\circ}, 180^{\circ}\right], \cos \lambda \leqslant 1$. For values of $i_{*}$ and $i_{p}$ on the interval $\left[0^{\circ}, 180^{\circ}\right], 1 \geqslant \sin i_{p} \geqslant 0$ and $1 \geqslant \sin i_{*} \geqslant 0$. Therefore, their product $\sin i_{*} \sin i_{p}$ is positive, and

$$
\sin i_{*} \sin i_{p} \cos \lambda \leqslant \sin i_{*} \sin i_{p} .
$$

Adding the term $\cos i_{*} \cos i_{p}$ to both sides gives

$$
\cos i_{*} \cos i_{p}+\sin i_{*} \sin i_{p} \cos \lambda \leqslant \cos i_{*} \cos i_{p}+\sin i_{*} \sin i_{p}
$$

Noting that the left side of Equation (8) is equal to $\cos \psi$ and making use of the identity $\cos (x-y)=\cos x \cos y+\sin x \sin y$ for the right side, it follows that

$$
\cos \psi \leqslant \cos \left(i_{*}-i_{p}\right)
$$

and therefore $\psi \geqslant i_{*}-i_{p}$. The same logic holds for $\psi \geqslant i_{p}-i_{*}$, so $\psi \geqslant\left|i_{*}-i_{p}\right|$. The absolute difference between the projected rotational inclination and the orbital inclination places a lower limit on the true spin-orbit angle $\psi$. A measurement of $i_{*}-i_{p}$ that is consistent with zero does not necessarily imply spin-orbit alignment, but a nonzero measurement of $i_{*}-i_{p}$ means that the system is misaligned by at least that value.

\section{ORCID iDs}

Brendan P. Bowler (i) https://orcid.org/0000-0003-2649-2288 Adam L. Kraus (iD https://orcid.org/0000-0001-9811-568X Marta L. Bryan (iD https://orcid.org/0000-0002-6076-5967 Matteo Brogi (iD https://orcid.org/0000-0002-7704-0153 Aaron C. Rizzuto (iD https://orcid.org/0000-0001-9982-1332 Andrew Vanderburg (iD https://orcid.org/0000-00017246-5438

Michael C. Liu (1D https://orcid.org/0000-0003-2232-7664 Lucas A. Cieza (iD https://orcid.org/0000-0002-2828-1153 


\section{References}

Allard, F., Homeier, D., \& Freytag, B. 2011, in ASP Conf. Ser. 448, 16th Cambridge Workshop on Cool Stars, Stellar Systems, and the Sun, ed. C. Johns-Krull, M. K. Browning, \& A. A. West (San Francisco, CA: ASP), 91

Allard, F., Homeier, D., \& Freytag, B. 2012, RSPTA, 370, 2765

Allers, K. N., \& Liu, M. C. 2013, ApJ, 772, 79

Allers, K. N., Jaffe, D. T., Luhman, K. L., et al. 2007, ApJ, 657, 511

Altmann, M., Roeser, S., Demleitner, M., Bastian, U., \& Schilbach, E. 2017, A\&A, 600, L4

Bailey, V., Hinz, P. M., Currie, T., et al. 2013, ApJ, 767, 31

Bailey, V., Meshkat, T., Reiter, M., et al. 2014, ApJL, 780, L4

Baraffe, I., Homeier, D., Allard, F., \& Chabrier, G. 2015, A\&A, 577, A42

Barman, T. S., Konopacky, Q. M., Macintosh, B., \& Marois, C. 2015, ApJ, 804,61

Basri, G., \& Batalha, C. 1990, ApJ, 363, 654

Bate, M. R. 2009, MNRAS, 392, 590

Batygin, K. 2012, Natur, 491, 418

Biller, B. A., Liu, M. C., Wahhaj, Z., et al. 2013, ApJ, 777, 160

Blunt, S., Nielsen, E. L., De Rosa, R. J., et al. 2017, AJ, 153, 229

Bochanski, J. J., West, A. A., Hawley, S. L., \& Covey, K. R. 2007, AJ, 133,531

Bonnefoy, M., Chauvin, G., Lagrange, A.-M., et al. 2014, A\&A, 562, A127

Booth, M., Jordan, A., Casassus, S., et al. 2016, MNRAS, 460, L10

Boss, A. P. 1997, Sci, 276, 1836

Boss, A. P. 2001, ApJL, 551, L167

Boss, A. P. 2006, ApJL, 637, L137

Bouvier, J., \& Appenzeller, I. 1992, A\&AS, 92, 481

Bouvier, J., Matt, S. P., Mohanty, S., et al. 2014, in Protostars and Planets VI, ed. H. Beuther et al. (Tucson, AZ: Univ. Arizona Press), 433

Bowler, B. P. 2016, PASP, 128, 102001

Bowler, B. P., Andrews, S. M., Kraus, A. L., et al. 2015, ApJL, 805, L17

Bowler, B. P., \& Hillenbrand, L. A. 2015, ApJL, 811, L30

Bowler, B. P., Liu, M. C., \& Cushing, M. C. 2009, ApJ, 706, 1114

Bowler, B. P., Liu, M. C., Kraus, A. L., \& Mann, A. W. 2014, ApJ, 784, 65

Bowler, B. P., Liu, M. C., Kraus, A. L., Mann, A. W., \& Ireland, M. J. 2011, ApJ, 743, 148

Bowler, B. P., Liu, M. C., Mawet, D., et al. 2017, AJ, 153, 18

Brandt, T. D., McElwain, M. W., Turner, E. L., et al. 2014, ApJ, 794, 159

Brinch, C., Jørgensen, J. K., Hogerheijde, M. R., Nelson, R. P., \& Gressel, O. 2016, ApJL, 830, L16

Brogi, M., Snellen, I. A. G., de Kok, R. J., et al. 2012, Natur, 486, 502

Bryan, M. L., Bowler, B. P., Knutson, H. A., et al. 2016, ApJ, 827, 100

Burrows, A., Marley, M., Hubbard, W. B., et al. 1997, ApJ, 491, 856

Caceres, C., Hardy, A., Schreiber, M. R., et al. 2015, ApJL, 806, L22

Cambrésy, L. 1999, A\&A, 345, 965

Cheetham, A. C., Kraus, A. L., Ireland, M. J., et al. 2015, ApJ, 813, 83

Christou, J. C., Neichel, B., Rigaut, F., et al. 2010, Proc. SPIE, 7736, 77361R

Chubak, C., Marcy, G., Fischer, D. A., et al. 2012, arXiv:1207.6212

Cieza, L., \& Baliber, N. 2007, ApJ, 671, 605

Cody, A. M., Hillenbrand, L. A., David, T. J., et al. 2017, ApJ, 836, 41

Cody, A. M., Stauffer, J., Baglin, A., et al. 2014, AJ, 147, 82

Cushing, M. C., Rayner, J. T., \& Vacca, W. D. 2005, ApJ, 623, 1115

Cushing, M. C., Vacca, W. D., \& Rayner, J. T. 2004, PASP, 116, 362

Cutri, R. M., et al. 2014, VizieR On-line Data Catalog, 2328, 0

Cutri, R. M., Skrutskie, M. F., van Dyk, S., et al. 2003, The 2MASS All-Sky Catalog of Point Sources (Univ. Massachusetts and Infrared Processing and Analysis Center, IPAC/California Institute of Technology), 2246, 0

Deacon, N. R., Schlieder, J. E., \& Murphy, S. J. 2016, MNRAS, 457, 3191

Dupuy, T. J., Liu, M. C., Bowler, B. P., et al. 2010, ApJ, 721, 1725

Evans, P. A., Osborne, J. P., Beardmore, A. P., et al. 2014, ApJS, 210, 8

Fabrycky, D. C., \& Winn, J. N. 2009, ApJ, 696, 1230

Fang, Q., Herczeg, G. J., \& Rizzuto, A. 2017, ApJ, 842, 123

Filippazzo, J. C., Rice, E. L., Faherty, J., et al. 2015, ApJ, 810, 158

Fitzpatrick, E. L. 1985, ApJ, 299, 219

Gaia Collaboration, Brown, A. G. A., Vallenari, A., et al. 2016, A\&A, 595, A2 Gauza, B., Bejar, V. J. S., Pérez-Garrido, A., et al. 2015, ApJ, 804, 96

Ginski, C., Schmidt, T. O. B., Mugrauer, M., et al. 2014, MNRAS, 444, 2280 Glebocki, R., \& Stawikowski, A. 1997, A\&A, 328, 579

Golimowski, D. A., Leggett, S. K., Marley, M. S., et al. 2004, AJ, 127, 3516 Gorlova, N. I., Meyer, M. R., Rieke, G. H., \& Liebert, J. 2003, ApJ, 593, 1074

Gotberg, Y., Davies, M. B., Mustill, A. J., Johansen, A., \& Church, R. P. 2016, A\&A, 592, A147

Hale, A. 1994, AJ, 107, 306
Harris, D. E., Forman, W., Gioa, I. M., et al. 1996, VizieR On-line Data Catalog: IX/13, 9013

Hartigan, P., Kenyon, S. J., Hartmann, L., et al. 1991, ApJ, 382, 617

Henden, A. A., Templeton, M., Terrell, D., et al. 2016, yCat, 2336, 0

Herbst, W., Bailer-Jones, C. A. L., Mundt, R., Meisenheimer, K., \& Wackermann, R. 2002, A\&A, 396, 513

Herczeg, G. J., \& Hillenbrand, L. A. 2014, ApJ, 786, 97

Holman, M., Touma, J., \& Tremaine, S. 1997, Natur, 386, 254

Horne, J. H., \& Baliunas, S. L. 1986, ApJ, 302, 757

Howell, S. B., Sobeck, C., Haas, M., \& Still, M. 2014, PASP, 126, 398

Ireland, M. J., Kraus, A., Martinache, F., Law, N., \& Hillenbrand, L. A. 2011, ApJ, 726, 113

Irwin, J., Berta, Z. K., Burke, C. J., et al. 2011, ApJ, 727, 56

Jensen, E. L. N., \& Akeson, R. 2014, Natur, 511, 567

Johnson, J. A., Winn, J. N., Albrecht, S., et al. 2009, PASP, 121, 1104

Kirkpatrick, J. D., Barman, T. S., Burgasser, A. J., et al. 2006, ApJ, 639, 1120

Konopacky, Q. M., Barman, T. S., Macintosh, B. A., \& Marois, C. 2013, Sci, 339, 1398

Konopacky, Q. M., Marois, C., Macintosh, B. A., et al. 2016, AJ, 152, 28

Kovács, G. 1981, Ap\&SS, 78, 175

Kozai, Y. 1962, ApJ, 67, 591

Kratter, K., \& Lodato, G. 2016, ARA\&A, 54, 271

Kraus, A. L., Andrews, S. M., Bowler, B. P., et al. 2015, ApJL, 798, L23

Kraus, A. L., Herczeg, G. J., Rizzuto, A. C., et al. 2017, ApJ, 838, 150

Kraus, A. L., \& Hillenbrand, L. A. 2008, ApJL, 686, L111

Kraus, A. L., Ireland, M. J., Cieza, L. A., et al. 2014, ApJ, 781, 20

Kraus, A. L., Ireland, M. J., Martinache, F., \& Lloyd, J. P. 2008, ApJ, 679, 762

Kraus, A. L., Tucker, R. A., Thompson, M. I., Craine, E. R., \& Hillenbrand, L. A. 2011, ApJ, 728, 48

Lachapelle, F.-R., LaFreniere, D., Gagné, J., et al. 2015, ApJ, 802, 61

Lafrenière, D., Jayawardhana, R., \& van Kerkwijk, M. H. 2010, ApJ, 719, 497

Lai, D. 2014, MNRAS, 440, 3532

Larkin, J., Barczys, M., Krabbe, A., et al. 2006, Proc. SPIE, 6269, 62691A

Lavigne, J.-F., Doyon, R., Lafrenière, D., Marois, C., \& Barman, T. 2009, ApJ, 704, 1098

Lee, K. I., Dunham, M. M., Myers, P. C., et al. 2016, ApJL, 820, L2

Leggett, S. K., Currie, M. J., Varricatt, W. P., et al. 2006, MNRAS, 373, 781 Lidov, M. L. 1962, P\&SS, 9, 719

Lomb, N. R. 1976, Ap\&SS, 39, 447

Low, C., \& Lynden-Bell, D. 1976, MNRAS, 176, 367

Lucas, P. W., Roche, P. F., Allard, F., \& Hauschildt, P. H. 2001, MNRAS, 326,695

Mace, G., Kim, H., Jaffe, D. T., et al. 2016, Proc. SPIE, 9908, 99080C

MacGregor, M. A., Wilner, D. J., Czekala, I., et al. 2017, ApJ, 835, 17

Mamajek, E. E. 2008, AN, 329, 10

Manjavacas, E., Bonnefoy, M., Schlieder, J. E., et al. 2014, A\&A, 564, A55

Mann, A. W., Gaidos, E., \& Ansdell, M. 2013, ApJ, 779, 188

Markwardt, C. B. 2009, in ASP Conf. Ser. 411, Astronomical Data Analysis Software and Systems XVIII, ed. D. A. Bohlender, D. Durand, \& P. Dowler (San Francisco, CA: ASP), 251

Martin, E. C., Mace, G. N., McLean, I. S., et al. 2017, ApJ, 838, 73

Matthews, B., Kennedy, G., Sibthorpe, B., et al. 2013, ApJ, 780, 97

McCaughrean, M. J., Close, L. M., Scholz, R.-D., et al. 2004, A\&A, 413, 1029

McElwain, M. W., Metchev, S. A., Larkin, J. E., et al. 2007, ApJ, 656, 505

McGovern, M. R., Kirkpatrick, J. D., McLean, I. S., et al. 2004, ApJ, 600, 1020

McGregor, P. J., Hart, J., Conroy, P. G., et al. 2003, Proc. SPIE, 4841, 1581

McLean, I. S., McGovern, M. R., Burgasser, A. J., et al. 2003, ApJ, 596, 561

Mieda, E., Wright, S. A., Larkin, J. E., et al. 2014, PASP, 126, 250

Monet, D. G., Levine, S. E., Canzian, B., et al. 2003, AJ, 125, 984

Montmerle, T., Koch-Miramond, L., Falgarone, E., \& Grindlay, J. E. 1983, ApJ, 269, 182

Muiños, J. L., \& Evans, D. W. 2014, AN, 335, 367

Naoz, S. 2016, ARA\&A, 54, 441

Naud, M.-E., Artigau, E., Malo, L., et al. 2014, ApJ, 787, 5

Offner, S. S. R., Dunham, M. M., Lee, K. I., Arce, H. G., \& Fielding, D. B. 2016, ApJL, 827, L11

Ohta, Y., Taruya, A., \& Suto, Y. 2005, ApJ, 662, 1118

Ortiz-León, G. N., Loinard, L., Kounkel, M. A., et al. 2017, ApJ, 834, 141

Park, C., Jaffe, D. T., Yuk, I.-S., et al. 2014, Proc. SPIE, 9147, 91471D

Pecaut, M. J., \& Mamajek, E. E. 2013, ApJS, 208, 9

Pecaut, M. J., \& Mamajek, E. E. 2016, MNRAS, 461, 794

Potter, D., Martín, E. L., Cushing, M. C., et al. 2002, ApJL, 567, L133

Queloz, D., Eggenberger, A., Mayor, M., et al. 2000, A\&A, 359, L13

Rameau, J., Nielsen, E. L., De Rosa, R. J., et al. 2016, ApJL, 822, L28

Ratzka, T., Köhler, R., \& Leinert, C. 2005, A\&A, 437, 611 
Rayner, J. T., Toomey, D. W., Onaka, P. M., et al. 2003, PASP, 115, 362

Rebull, L. M., Wolff, S. C., \& Strom, S. E. 2004, AJ, 127, 1029

Reggiani, M., Meyer, M. R., Chauvin, G., et al. 2016, A\&A, 586, A147

Reidemeister, M., Krivov, A. V., Schmidt, T. O. B., et al. 2009, A\&A, 503,247

Reinhold, T., \& Gizon, L. 2015, A\&A, 583, A65

Rizzuto, A. C., Ireland, M. J., \& Kraus, A. L. 2015, MNRAS, 448, 2737

Rizzuto, A. C., Ireland, M. J., \& Robertson, J. G. 2011, MNRAS, 416, 3108

Rucinski, S. 1999, in ASP Conf. Ser. 185, IAU Colloq. 170, Precise Stellar Radial Velocities, ed. J. B. Hearnshaw \& C. D. Scarfe (San Francisco, CA: ASP), 82

Scargle, J. D. 1982, ApJ, 263, 835

Schmidt, T. O. B., Neuhäuser, R., Seifahrt, A., et al. 2008, A\&A, 491, 311

Scholz, R.-D., Mccaughrean, M. J., Lodieu, N., \& Kuhlbrodt, B. 2003, A\&A, 398, L29

Schwarz, H., Ginski, C., de Kok, R. J., et al. 2016, A\&A, 593, A74

Seifahrt, A., Neuhäuser, R., \& Hauschildt, P. H. 2007, A\&A, 463, 309

Slesnick, C. L., Hillenbrand, L. A., \& Carpenter, J. M. 2004, ApJ, 610, 1045

Stamatellos, D., \& Herczeg, G. J. 2015, MNRAS, 449, 3432

Stauffer, J., Cody, A. M., Rebull, L., et al. 2016, AJ, 151, 60
Todorov, K., Luhman, K. L., \& Mcleod, K. K. 2010, ApJL, 714, L84

Vacca, W. D., Cushing, M. C., \& Rayner, J. T. 2003, PASP, 115, 389

Vanderburg, A., \& Johnson, J. A. 2014, PASP, 126, 948

Voges, W., Aschenbach, B., Boller, T., et al. 2000, IAUC, 7432, 3

Vogt, S. S., Allen, S. L., Bigelow, B. C., et al. 1994, Proc. SPIE, 2198, 362

Wilking, B. A., Gagné, M., \& Allen, L. E. 2008, in Handbook of Star Forming Regions, Vol. II, ed. B. Reipurth (San Francisco, CA: ASP), 351

Wilking, B. A., Vrba, F. J., \& Sullivan, T. 2015, ApJ, 815, 2

Williams, J. P., Mann, R. K., Francesco, J. D., et al. 2014, ApJ, 796, 120

Winn, J. N., Noyes, R. W., \& Holman, M. J. 2005, ApJ, 631, 1215

Wizinowich, P. 2013, PASP, 125, 798

Wolff, S. G., Menard, F., Caceres, C., et al. 2017, AJ, 154, 26

Wu, Y.-L., Close, L. M., Males, J. R., et al. 2015a, ApJ, 801, 4

Wu, Y.-L., Close, L. M., Males, J. R., et al. 2015b, ApJL, 807, 1

Wu, Y.-L., Sheehan, P. D., Males, J. R., et al. 2017, ApJ, 836, 223

Wyatt, M. C. 2008, ARA\&A, 46, 339

Yelda, S., Lu, J. R., Ghez, A. M., et al. 2010, ApJ, 725, 331

Zhou, Y., Herczeg, G. J., Kraus, A. L., Metchev, S., \& Cruz, K. L. 2014, ApJL, 783, L17

Zucker, S. 2003, MNRAS, 342, 1291 